\title{
El significado de la Teoría Política: gestión de cambios estructurales e innovación política*
}

\author{
Ricard Zapata Barrero \\ Universitat Pompeu Fabra \\ Grup de Recerca de Teoria Política (GRTP) \\ ricard.zapata@upf.es
}

RESUMEN

Los cambios tan rápidos de parámetros que estamos viviendo estos últimos años están confrontando a los investigadores científicos sociales a nuevos contextos y perspectivas. Son situaciones tan nuevas que la tarea conceptual sobre cómo abordar estos nuevos interrogantes se torna una necesidad para la propia dinámica innovadora de la disciplina. Lo cierto es que cada vez más se tiene la convicción de que las reflexiones teóricas y las decisiones políticas que se tomen aquí y ahora tienen una importancia vital, puesto que lo que se está diseñando es la sociedad política del futuro. En este marco, la Teoría Política (TP) como actividad crítica tiene un campo de cultivo sin precedentes en nuestra historia más reciente. Se trata de plantear dos preguntas vinculadas entre sí: ¿Cuál es su función en relación con esta realidad de inestabilidad social y política? ¿Cuáles son los instrumentos (conceptos, enfoques, etc.) que nos puede proporcionar para el análisis de estos cambios estructurales? Articularé mi razonamiento en seis secciones. En la primera sección presento los primeros elementos para iniciar una reflexión sobre la TP hoy en día. En la segunda sección me planteo cuál es el mirador desde el cual el teórico político interpreta la realidad cambiante, centrándome especialmente en su unidad de análisis y objetivos básicos. La tercera sección es histórica. Repaso las principales etapas de la TP, y centrándome en la última de acuerdo con mi hilo conductor: el análisis de los contextos de cambios sociales y políticos. Me planteo luego, en una cuarta sección, cuáles son los principales programas de investigación que actualmente contribuyen al estudio de estos cambios estructurales. Para completar este argumento, en una quinta sección, profundizo los principales enfoques que existen para analizar estos cambios, los cuales determinan formas diferentes de concebir la actividad propia de la TP. Por último, en una sexta y última sección, vuelvo a tomar el significado de la TP hoy en día, señalando sus principales retos y expectativas, como gestora de innovación política. Acabo con unas reflexiones para dejar abierto este programa de investigación. En un apéndice final presento unos primeros apuntes sobre la TP en España, como base para una investigación futura.

Palabras clave: Teoría Política, Evolución Histórica, Programas de Investigación, Procesos de Cambio, Innovación Política.

\footnotetext{
* Agradezco los comentarios y críticas recibidos en versiones anteriores de este artículo de los miembros del Grup de Recerca de Teoria Política (GRTP) de la UPF y de los evaluadores de REIS. Unas versiones más reducidas de este artículo han aparecido en la revista Claves («La actualidad de la Teoría Política», 135, 2003: 38-43) y en European Political Science («Political theory today: political innovation and the management of structural change», Summer 2004).
} 


\section{INTRODUCCIÓN: RAZONES DE UN BALANCE}

Estamos en una época de gran turbulencia, de conflictos y de dudas sobre nuestros propios marcos de referencia políticos, de confusión sobre el presente y de desorientación hacia el futuro. Los cambios tan rápidos de parámetros que estamos viviendo estos últimos años están confrontando a los investigadores científicos sociales a nuevos escenarios y perspectivas. Son situaciones tan nuevas que la tarea conceptual sobre cómo abordar estos nuevos contextos se torna una necesidad para la propia dinámica innovadora de la disciplina. Lo cierto es que cada vez más se tiene la convicción de que las reflexiones teóricas y las decisiones políticas que se tomen aquí y ahora tienen una importancia vital, puesto que lo que se está diseñando es la sociedad política del futuro. En este marco, la Teoría Política (TP) como actividad crítica tiene un campo de cultivo sin precedentes en nuestra historia más reciente. Se trata de plantear dos preguntas vinculadas entre sí: ¿Cuál es su función en relación con esta realidad de inestabilidad social y política? ¿Cuáles son los instrumentos (conceptos, enfoques, etc.) que nos puede proporcionar para el análisis de estos procesos de cambio?

Articularé mi razonamiento en seis secciones. En la primera sección presento los primeros elementos para iniciar una reflexión sobre la TP hoy en día. En la segunda sección me planteo cuál es el mirador desde el cual el teórico político interpreta la realidad cambiante. La tercera sección será histórica. Repaso las principales etapas de la TP, y amplío la última de acuerdo con mi hilo conductor: la actualidad de la TP para el análisis de los contextos de cambios sociales y políticos. Me planteo luego, en una cuarta sección, cuáles son los principales programas de investigación que actualmente contribuyen al estudio de estos cambios estructurales. Para completar este argumento, en una quinta sección, profundizo los principales enfoques que tenemos para analizar estos cambios, los cuales determinan formas diferentes de concebir la actividad propia de la TP. Por último, en una sexta y última sección, vuelvo a tomar el significado de la TP hoy en día, señalando sus principales retos y sus expectativas futuras, como gestora de procesos de cambio y principal fuente de innovación política. Acabo con unas reflexiones finales para dejar abierto este programa de investigación propuesto.

\section{TEORIZANDO SOBRE LA TEORÍA POLÍTICA}

En febrero de 1939, G. H. Sabine escribió un artículo titulado «What is a Polítical Theory?»; en agosto del 2002, S. White, el director de Political Theory, dedica un monográfico precisamente sobre esta cuestión, "What is Political Theory?», siguiendo dos líneas de análisis: «Traditional Resources, Novel Challenges» y «Political Theory and Political Science». Entre 
estas dos fechas han pasado sesenta y tres años. Podríamos considerar que éste es el período que tiene la reflexión acerca de la tarea de la TP. Confieso, de entrada, que la pregunta acerca de ¿Qué es...? me incomoda tanto por su forma ontológica como porque me invita a dar respuestas esencialistas. Son preguntas más propias de filósofos que de teóricos políticos. En estas páginas plantearemos más bien una pregunta simple:¿cómo reconocemos una actividad académica como siendo de Teoría Política? Para poder responder a estas preguntas se requiere analizar actividades y contextos reales.

Al iniciar una reflexión dentro de estos primeros límites debemos hacer frente a dos disyuntivas. La primera se centra en determinar su lugar dentro de las Ciencias Políticas $(C P)$. A saber, si la TP es una subdisciplina de la $C P$ o bien una actividad distintiva, un campo propio de la $C P^{1}$. La segunda disyuntiva se interesa principalmente por determinar cuál es su función en relación con la sociedad y la actividad política. Esto es, si la TP debe tener un objetivo estrictamente interpretativo o bien también aplicado, directamente ejerciendo una influencia sobre la realidad social y los que deciden políticas ${ }^{2}$.

La concepción que aquí seguiré es que la TP es una actividad independiente, que tiene básicamente una función aplicada. Sin voluntad aplicada la reflexión teórica carece de sentido. De hecho, como tendré ocasión de insistir, el único significado que tiene la interpretación teórica reside en su carácter clarificador de las confusiones conceptuales y desorientaciones prácticas que generan los procesos de cambio 3 .

Asimismo, existe un segundo tipo de problema que requiere resolverse para iniciar una reflexión sobre la TP hoy en día. La confusión implícita que existe entre el objeto y el contexto dentro del cual se realiza la actividad. Se suele dar por implícito que la TP es reflexión sobre la Democracia y el Liberalismo. Podemos decir que la TP está muy estrechamente vinculada al modo de vida de una comunidad concreta, y que su función consiste en articular la autocomprensión de dicha comunidad. Si entendemos por comunidad precisamente el sistema liberal democrático, tendremos un argumento contextual sólido de por qué la TP es hoy en día básicamente Teoría de y sobre la Democracia y el Liberalismo.

\footnotetext{
1 Ésta es la dicotomía que guía el enfoque de J. G. Gunnell (1983), el cual precisamente resuelve de forma totalmente contraria a la de D. Miller y L. Sidentop (1983), que es la que seguiremos aquí.

2 Dicotomía establecida por A. Vincent (1997). Véanse también a D. Held y A. Leftwich (1987).

3 Véase, entre otros, el interesante monográfico dedicado a la gestión de las transformaciones sociales (management of social transformations) de la International Political Science Review, editada por K. Wiltshire (2001). Véase, asimismo, una aplicación de este argumento en el contexto de las migraciones internacionales en G. Aubarell y R. Zapata Barrero (eds., 2003), así como un estudio de los procesos de cambio que se están produciendo en España con la llegada de inmigrantes (R. Zapata Barrero, 2004b).
} 
Aunque no parezca oportuno acabar con una evidencia, la TP es teorización sobre la Política, es decir, reflexión y actividad de segundo orden. Teniendo esta premisa como trasfondo, podemos considerar tres niveles de análisis:

Nivel 1 Reflexión teórica sobre la relación entre las instituciones políticas y los ciudadanos. Análisis normativo de los marcos institucionales.

Nivel 2 Reflexión sobre aquellos que estuvieron en el primer nivel en el pasado. Historia de la Teoría Política. Análisis de las tradiciones históricas, de los conceptos políticos y de sus principales representantes.

Nivel 3 Meta-teoría política. Reflexión de tercer orden. Sobre metodología y técnicas de investigación para llevar a cabo los dos primeros niveles. Este tercer nivel de análisis puede ser de dos tipos correspondientes a cada uno de los dos niveles anteriores (Nivel 3/1, Nivel 3/2).

El enfoque que seguiré en estas páginas vincula el Nivel 3 con el Nivel $1^{4}$.

\section{UN MIRADOR PARA EL ANÁLISIS DE CONTEXTOS DE CAMBIOS}

La TP debe ser considerada como una actividad, una condición, diría incluso como una actitud y un estado de consciencia, siempre de segundo orden. Trata de dar sentido a los cambios políticos y es esa búsqueda de significado la que caracteriza su práctica. Esto implica que al comenzar a hablar de la TP nos debemos enfrentar con numerosas dificultades que tienen la forma de una paradoja, puesto que circunscribir la tarea de la TP está lleno de problemas teóricos. Casi todos los autores que practican la TP y que en algún momento dado reflexionan sobre «lo que hacen» (estando en Nivel 1, pasan a Nivel 3$)^{5}$ compartirían las dificultades que existen para delimitar tanto las unidades de análisis como los objetivos propios de la $T P^{6}$.

4 Existe también un debate muy activo que vincula el Nivel 3 con el 2 que dejamos de lado. Las grandes «centrales eléctricas» de estos debates las constituyen los representantes de la escuela de Cambridge, como Q. Skinner (1969, 1970, 1974), J. G. A. Pocock (1981, 1982,1985). También son imprescindibles J. G. Ch. D. Tarlton (1973), A. Lockyer (1979), Gunnell (1979, 1982), D. Lacapra (1980), D. Lacapra y S. L. Kaplan (eds., 1982), J. S. Nelson (ed., 1986), D. R. Kelley (1987), M. Bevir (1999), K. Palonen (2002). Así como los de tradición alemana, empezando por R. Koselleck (1993), M. Richter (1995). Entre nosotros, véanse los trabajos seminales de F. Vallespín (1990, 1992).

5 Existen pocos trabajos que se hayan planteado la TP como disciplina. Existen reflexiones dispersas en artículos, capítulos e introducciones de libros, pero no una obra propia que podamos considerar como «libro de referencia». Uno de los únicos que podría recibir tal consideración es la edición de D. Miller y L. Siedentop (eds., 1983). Como programa de investigación es sobre todo en los años noventa cuando aparecen los primeros trabajos. Podemos destacar, entre los más conocidos, a D. Held (1991), R. Bellamy (1993), A. Vincent (1997), T. Ball (1995), N. P. Barry (1995; la primera edición es de 1979), Ph. Pettit (1991), K. von Beyne (1994).

6 Véanse, por ejemplo, el prefacio a la primera edición y las palabras introductorias de N. P. Barry (1995). 
Por ejemplo, mantener que las principales unidades de análisis de la TP son el Estado y el Poder, como lo hace D. Held (1991: 5 ss.), y que la mayoría de las TP se sitúan en una de estas dos categorías, es demasiado confuso, puesto que estas unidades también caracterizan a la $C P$ como disciplina. También podríamos decir que la principal unidad de análisis de la TP es el conflicto o los valores, pero de nuevo caeríamos en la misma confusión. Es cierto que la TP se interesa, como campo propio de la CP que es, por el Estado, el Poder, el Conflicto, los Valores. Pero al considerar estos elementos como siendo propios incurriríamos en una confusión entre objeto y contexto (considerando la CP como contexto). Creo que el mejor procedimiento para poder identificar una unidad de análisis propia de la TP es plantearnos algo así como una "guía para la práctica de la $T P$ »: ¿qué elementos destacaríamos como necesarios? De entrada, dividiría esta supuesta «guía» en, al menos, tres secciones: i) el tipo de actividad que supone; ii) las premisas epistemológicas (o convicciones) que debe tener toda actividad teórica, y iii) por último, pero no menos importante, el contexto dentro del cual se desarrolla dicha actividad. Déjenme ampliar brevemente cada una de estas secciones.

i) Mi argumento es que la TP es un tipo de actividad de segundo orden eminentemente analítica. Esto significa al menos tres cosas. Por un lado, su tarea consiste en cuestionar lo que se da por sentado y/o en obligar a definir aquello que se da por implícito tanto para apoyar argumentos como para legitimar actividades e instituciones. Por otro lado, hacer TP es buscar siempre el sistema de «prejuicios» (en el sentido de H. G. Gadamer, 1992) que acompaña la realidad política y orienta su actividad (B. Parekh, 1968). Por último, y como corolario, la actividad de la TP siempre tiene un efecto espejo. Este hecho es para mí de suma importancia. Este efecto espejo significa que las actividades de la TP siempre obligan a tener en cuenta el marco de referencia dentro del cual se lleva a cabo la argumentación. En definitiva, como actividad analítica, es el resultado del Giro Lingüístico aplicado a la reflexión sobre la política. Esto significa básicamente que constantemente debe ser autoconsciente del lenguaje que se usa en política (T. D. Weldon, 1953; N. P. Barry, 1995: 7).

ii) Se sigue que epistemológicamente hacer TP implica un doble abandono: la pretensión a la Objetividad, en el sentido conductista (Ch. Taylor, 1985), y la tendencia en percibir la realidad y las proposiciones en términos de verdad o falsedad, en el sentido positivista. En el lenguaje de Gadamer, diríamos que el que hace TP debe saber que no puede escapar al "círculo de la comprensión», y que sus mismas proposiciones y mismo lenguaje pueden (y deben) ser objeto de análisis. En este marco, su principal tarea es desmitificar conceptos o ideas asentados. Reconocer que los conceptos que se usan dependen siempre de criterios valorativos, y son, por lo tanto, esencialmente polémicos (W. Gallie, 1955; W. Connolly, 1974).

iii) Finalmente, lo propio de la TP es reflexionar sobre cómo se articula la relación entre los conceptos políticos y la estructura cambiante de la sociedad (D. Miller y L. Siedentop, 
1983: 1$)^{7}$. Esto explica que el marco contextual idóneo para practicar TP es en períodos de crisis, de dudas sobre el statu quo, de cambios sociales. Esto podría explicar, por ejemplo, que la TP quedara en segundo plano durante el período conductista (años cincuentasetenta), puesto que política y socialmente fue un período de estabilidad y de consenso. En términos kuhnianos, podríamos decir que la TP tiene su campo de cultivo en períodos «revolucionarios». Esto es, en momentos históricos donde los paradigmas tradicionales que conforman las concepciones del mundo y limitan todo proceso de legitimación política, comienzan a mostrar sus primeras «incoherencias».

Invirtiendo el argumento, cuando el desarrollo de la sociedad y de la política es normal (en sentido kuhniano) no hay necesidad imperiosa de la TP. Se sigue, pues, y como corolario, que la TP cobra su sentido cuando se produce una distancia entre el vocabulario y la realidad que pretende describir. Esto es, cuando ya no disponemos de recursos conceptuales para legitimar un contexto de inestabilidad. Desde este punto de vista, la TP es una actividad que pretende dar sentido y orientaciones en tiempos de confusiones, pero también tiene una función innovadora de búsqueda de nuevos conceptos y orientaciones prácticas en un período obligado de abandonar parte de lo que se había creído antes ${ }^{8}$. Siguiéndonos basando en las aportaciones de T. Kuhn, el contexto de la TP puede describirse como un lugar donde abundan unas situaciones de inconmensurabilidad y de incoherencias ${ }^{9}$. La TP siempre busca paradojas, incongruencias, inconsistencias entre la teoría y la práctica, entre marcos de referencias y actividades. En otros términos, la TP no sólo se ocupa de identificar problemas, desafíos y retos que ponen en entredicho el marco de referencia dentro del cual se legitima la actividad política, sino que también su tarea quedaría incompleta si no ofreciese vías para sus posibles soluciones (ésta es la función aplicada, que trataremos más adelante).

De todo lo anterior podemos concluir que la TP no puede, por lo tanto, escapar del contexto. La primera ley de hierro es, en este sentido, que existe un vínculo estrecho entre la práctica de la TP y el contexto de conflicto y de inestabilidad que pretende analizar. Sin conflicto no hay problemas, sin problemas no hay preguntas, y sin preguntas no hay el primer gran ingrediente de la tarea teórica política. La TP no sólo es método, no sólo es construcción de teoría y reflexión sobre conceptos. La TP está sobre todo guiada por problemas (hacia esa dirección parece inclinarse I. Shapiro, 2002). Una buena reflexión teórica empieza por constataciones iniciales y por identificar problemas y conflictos. Mi argumento es

\footnotetext{
7 Ésta es la Unidad de Análisis del último trabajo de R. Zapata Barrero (ed., 2005).

8 Ésta es una de las diferencias entre desarrollo normal y revolucionario de Kuhn (1962, por ejemplo; 2000: 121).

9 Para Kuhn, las «incoherencias» son un detector de cambio revolucionario (2000: 41), y la «inconmensurabilidad», una situación extrema donde se produce un divorcio radical entre conceptos/lenguaje y la realidad que le da sentido (véase, por ejemplo, T. Kuhn, 2000, cap. 2: 47-75).
} 
que los conflictos que le interesan son estructurales, los que ponen en duda el mismo statu quo y marcos de referencia tradicionales.

Para llevar a cabo esta actividad, la TP utiliza, por separado o en combinación, instrumentos propios: argumentos, valores, conceptos, principios y criterios, tradiciones. Si miramos los índices de los libros de $T P$, veremos que la mayoría de los elementos que articulan su universo son discusiones acerca de valores y principios (justicia, igualdad, libertad, derechos humanos), conceptos (poder, autoridad, obligación, control social) y tradiciones (marxismo, republicanismo, comunitarismo, liberalismo $)^{10}$.

Estos recursos pueden servir para dos objetivos básicos: interpretar y/o actuar e intervenir sobre la realidad y participar en el proceso de cambio social y político. Estos dos objetivos básicos ilustran dos concepciones existentes de la TP: interpretar y comprender la realidad política, y/o intervenir en ella, como TP aplicada. En la introducción ya me he pronunciado respecto a esta disyuntiva. Ahora detallaré algo más en qué consiste cada objetivo y me inclinaré por combinarlos en lugar de tomarlos como objetivos independientes ${ }^{11}$. Denominaré al primer objetivo concepción hermenéutica de la actividad de la TP; y al segundo objetivo, concepción aplicada. Asimismo, sostendré que detrás de la actividad hermenéutica se defienden principios teóricos, mientras que la concepción aplicada se guía por principios prácticos.

La concepción hermenéutica nos dice que el objetivo propio de la TP es básicamente interpretar, intentando desligar los hechos de los valores que le rodean, identificar el sistema de creencias y de supuestos, pero sin sobrepasar el análisis descriptivo y explicativo. Parte del supuesto de que no es posible una interpretación neutra, sino que toda interpretación está ligada a valores, ideologías y tradiciones. Que la tarea misma de la interpretación es una actitud inevitable ${ }^{12}$. Su objetivo último es enumerar una serie de principios teóricos que sirvan para entender y/o evaluar la realidad política, y orientar y/o criticar la práctica.

La concepción aplicada parte de la convicción de que un buen teórico político debe siempre buscar tener un impacto sobre la realidad, modificándola y mejorándola ${ }^{13}$. El argumento es

\footnotetext{
10 Véanse, entre otros, el ya clásico manual de referencia de F. Vallespín (coord., 1992-1995), R. del Águila, F. Vallespín y otros (1998), y los de reciente aparición en España, como R. Máiz (comp., 2001), J. Antón (coord., 2002).

11 Me basaré en la diferencia de A. Vincent (1997: 5 ss.) entre concepción inclusiva (interpretativa) y concepción exclusiva (aplicada) de la TP.

12 En este marco de la discusión son sugestivas las declaraciones de T. Ball cuando nos dice que «the decision to interpret or not to interpret is not an option open to human beings» (1995: 7).

13 Por parafrasear las palabras de R. del Águila en la Introducción de la monografía de la Revista Española de Ciencia Política dedicada a la Teoría Política, «la vocación de la teoría política no es (en realidad nunca fue) vivir al margen del mundo, sino intervenir en él» (R. del Águila, 2000: 8).
} 
el siguiente: si admitimos que su actividad hermenéutica se realiza sobre todo en contextos de «turbulencia», esta interpretación sólo adquiere sentido si ayuda a orientar este cambio y si participa en él. Los principios que resulten de su actividad deben ser, en este sentido, prácticos. Según esta concepción, la TP busca proporcionar marcos de referencia para las acciones y decisiones políticas, ofrecer esquemas conceptuales para legitimar instituciones, principios prácticos para los que «hacen política» (políticos, asociaciones, partidos, etc.). En un monográfico de la revista Dissent dedicado a hacer un estado de la cuestión de la TP, M. Walzer, su director, nos dice en la Introducción que sin esta vocación a tener un practical impact la TP se convertiría en «endless refinement, esoteric jargon, romantic posturing, and fierce intramural polemic» (M. Walzer, 1989: 337). En la misma línea se sitúa Ch. Taylor, para quien la TP no puede estar divorciada de la political action (Ch. Taylor, 1983).

Lo propio de la TP es saber combinar estas dos concepciones. La concepción hermenéutica carece de sentido si no ofrece instrumentos de aplicación; la concepción aplicada carece de orientación sin una base interpretativa sólida. En este sentido, tras un análisis interpretativo que destaca principios teóricos, su función es dar recomendaciones a través de principios prácticos ${ }^{14}$. La tarea de «traducir» los principios teóricos en principios prácticos es uno de los principales momentos de la actividad de la TP. El teórico político, en el fondo, no es más que un intérprete y traductor, un gestor de los desideratums de la sociedad.

\section{EVOLUCIÓN HISTÓRICA: INICIOS, INCERTIDUMBRE Y CONSOLIDACIÓN}

Si queda todavía por hacer un obra de referencia que haga un estado de la cuestión de la $T P$, aún más justificado sería hacer una historia de la TP, siguiendo, por ejemplo, las mismas intenciones de obras ya de referencia como la de J. Farr y R. Seidelman (eds., 1993) o la de J. Farr, J. S. Dryzek y S. T. Leonard (eds., 1995) ${ }^{15}$. Para iniciar esta empresa sería muy interesante poder editar una recopilación de textos (un Reader) sobre «estados de cuestión» de la TP que han aparecido a lo largo del siglo $x^{16}$. Sin duda, tendríamos una prime-

\footnotetext{
${ }^{14}$ En esta línea se manifiesta la dimensión normativa que trataremos más adelante. Por ejemplo, la contribución de B. Hindess (1997) en la obra editada por A. Vincent incide en este aspecto.

15 Aunque se pediría, eso sí, que fueran menos centradas en la evolución de la disciplina en Estados Unidos.

16 Desde el artículo de G. H. Sabine (1939) hacia delante hasta llegar a la edición especial de Political Theory (S. White, ed., 2002). Aunque no pretendo ser exhaustivo, sino ilustrativo, para reforzar la necesidad de hacer una obra que los compile, este supuesto Reader debería, sin duda, contener los siguientes trabajos: J. Plamenatz (1960), I. Berlin (1962), B. Parekh (1968), S. Wolin (1969), B. Barry (1980), B. Barber (1980), J. G. Gunnell (1983), D. Miller (1990), además de algunos capítulos de las siguientes obras: D. Miller y L. Siedentop (eds., 1983), J. G. Gunnell (1986), Ph. Pettit (1991), D. Held (ed., 1991), A. Vicent (ed., 1997), R. Bellamy (1993), N. P. Barry (1995), T. Ball (1995). Una obra recopilatoria de estas características está todavía por hacer. Su utilidad para la docencia y la investigación está fuera de duda.
} 
ra obra que trazara las discusiones que se han producido, útil para aquellos interesados en conocer la evolución de la disciplina. Constataríamos, y éste será el argumento que me guiará, que su marco de reflexión se centra en los cambios y no tanto en los elementos que permanecen.

Esta sección la planteo como un ejercicio de presentación de un esquema que justificara la estructura que podría tener dicha obra. Existen dos formas principales de enfocar una evolución histórica de la TP. O bien seguimos como hilo conductor las diferentes etapas de su relación con la $C P$, la historia de la $T P$ como disciplina es la historia de su relación con la CP (éste es el enfoque seguido, entre otros, por J. C. Gunnell, 1983), o bien seguimos los acontecimientos históricos que sin duda influyen en su orientación. En esta sección combinaremos estos dos enfoques. Seguiremos el primero hasta los años setenta, y destacaremos, de acuerdo con nuestra línea de análisis, algunos hechos históricos que orientan los debates a partir de los setenta.

En general, podemos distinguir tres etapas históricas que van desde finales de 1930 hasta nuestros días. Una etapa de inicios de 1930 a 1945, una etapa de incertidumbre de 1945 a 1970 y, finalmente, una etapa de consolidación que comienza en los años setenta. Ampliaré sobre todo las dos últimas etapas.

\subsection{Inicios: $1930-1945$}

Esta primera etapa se sitúa en el período de entreguerras. La TP se percibe básicamente como historia del pensamiento político y análisis conceptual. El paso de confirmación es, sin duda, la conocida Historia de la Teoría Política, de G. Sabine y T. Thorson (1937), justo dos años antes de su artículo, de 1939, «What is a Political Theory?». En este artículo, Sabine insiste en que la TP sólo puede describirse como disciplina cuando se basa principalmente en tres tipos de proposiciones: las proposiciones basadas en hechos o proposiciones factuales, las basadas en explicaciones o proposiciones causales, y las estrictamente cargadas de valores o proposiciones evaluativas. Sugería la necesidad de distinguir, pero al mismo tiempo vincular, estos tres aspectos en el momento de hacer tanto historia de la TP (Nivel 2) como análisis contemporáneo (Nivel 1).

En este período se admite que el vínculo entre el análisis conceptual y su dimensión histórica es una de las primeras formas que adopta la TP como disciplina. Siguiendo las distinciones introducidas al principio, la TP comienza en el Nivel 2, como Historia de la $T P$, especialmente centrada en la relación entre conceptos/tradiciones/pensadores políticos. Esto es, analiza la carga semántica que tienen los conceptos políticos cuando son 
usados por los pensadores a través de la historia. Es importante señalar que este tipo de análisis de los conceptos percibe la historia como historia de las tradiciones políticas y corrientes de pensamiento. En esta línea se interesa sobre todo no sólo por el uso de conceptos políticos, sino por su relación con las tradiciones y los contextos. De hecho, el mismo Sabine vincula muy estrechamente la producción teórica con los contextos de crisis:

«Political Theories are secreted [...] in the interstices of political and social crisis. They are produced, not indeed by the crisis as such, but by its reaction on minds that have the sensitivity and the intellectual penetration to be aware of crisis» (Sabine, 1939: 3).

En cualquier caso, en estos años uno situaba la TP como expresando un interés acerca de las ideas y de los conceptos relacionados con la política (Gunnell, 1983: 7). El mismo Gunnell insiste en que este período de cristalización de la Historia de la Teoría Política como nuevo campo de investigación tiene unos factores explicativos contextuales. Los primeros pasos de la TP comienzan en un contexto que necesita demostrar los valores positivos del liberalismo frente al comunismo y al fascismo emergentes, como pensamientos políticos basados en su anti-liberalismo.

\subsection{Incertidumbre: $1945-1970$}

La segunda etapa es la que coincide con la fase conductista (1950-70). Casi toda la literatura comparte la convicción de que apenas hay TP por dominio absoluto del positivismo lógico. La TP se percibe meramente como reflexión sobre métodos en el sentido cientifista del término. Es decir, discusiones sobre procedimientos epistemológicos para llevar a cabo una tarea metodológica en el sentido pretendido por el conductismo. La TP era, pues, vista como teoría científica acerca de los problemas que el investigador encuentra para llevar a cabo sus tareas científicas. Aunque no debemos olvidar que la así llamada «revolución conductista» debe considerarse como una «revolución teórica»17. La TP durante estos años se debate entre ser considerada como "carente de sentido», en el sentido positivista, por ser histórica y moralista, o bien como formando parte de las reflexiones que se plantea el investigador conductista, como TP empírica. De estos dos extremos -el histórico y moralista y el metodológico propiamente dicho-, el conductismo proclamó el declive del primer polo.

17 En esta línea, A. C. Gunnell (1983: 13) afirma que los grandes representantes de dicha revolución eran what they sought to replace, es decir, teóricos políticos en el sentido histórico y normativo (D. Easton, R. Dahl, K. Deutsch, entre otros). 
El segundo extremo era el que prevaleció como TP, como preocupación por lo que es, y no por lo que debe ser. Será en el proceso de configuración de un frente contra el conductismo reinante, a finales de los sesenta, cuando la TP vuelve a producir literatura. Por ejemplo, en la línea de Sabine, aparece el Politics and Vision de S. Wolin (1960). En dicha obra, Wolin indica que la TP equivale al estudio de las grandes tradiciones históricas. Casi al mismo tiempo que en diferentes momentos de finales de los cincuenta y a lo largo de los sesenta se formulaban argumentos en contra de la TP o simplemente se postulaba su desaparición ${ }^{18}$, aparecen dos trabajos que, podríamos decir, desde la «primera línea de combate» se «atrevieron» a contra-argumentar el discurso hegemónico: uno es de S. Wolin (1969) y el otro de B. Parekh (1968). A mi conocimiento, estos dos trabajos contribuyen de forma independiente a cerrar este período.

No es causalidad que el artículo de S. Wolin (1969) apareciera en el mismo número de la American Political Science Review que el famoso trabajo de D. Easton (1969), quien proclama el inicio de la etapa posconductista. En él, S. Wolin, y siguiendo la misma línea crítica de D. Easton, se dirige contra el exceso de una de las principales banderas del conductismo: el «metodismo». Este supuesto triunfo del metodismo hace perder a la $C P$ el sentido de la realidad ${ }^{19}$. Asimismo, se esfuerza por separar el discurso sobre el método del discurso sobre la teoría, dos enfoques que los conductistas tienden a confundir y a tomar el uno por el otro. Al final de su trabajo, S. Wolin (1969: 1077 ss.) insiste en que la TP como vocación se distingue, entre otras cosas, porque dota al conocimiento de la política de una dimensión histórica que le fue negada por el cientifismo conductista.

En este momento de su razonamiento, Wolin sugiere que la aplicación de esta sensibilidad histórica a la reflexión política significa, al menos, tres cosas: por un lado, lo que podríamos denominar su capacidad analítica, esto es, el hecho que al interpretar la política se intenta dar sentido a una realidad que en la práctica es compleja. La tarea de la $T P$ es «to grasp present structures and interrelationships, and to re-present them in a new way» (Wolin, 1969: 1078). En segundo lugar, su preocupación por la cosa pública (res publica). La TP es preocupación por la esfera y las relaciones públicas. En tercer lugar, el hecho que se interese por acontecimientos que suponen crisis y cambios sociales y políticos.

18 Que la TP había desaparecido, o incluso «muerto», en los años sesenta aparece en muchos autores: A. Cobban (1953), P. Laslett (1956), I. Berlin (1962), D. Easton (1953), R. Dahl (1958). No es causalidad que estas constataciones se hagan en pleno período de «euforia conductista».

19 Resulta indicativo que esta crítica al metodismo de la CP es la que articula también el reciente artículo de I. Shapiro (2002) en su contribución al monográfico de Political Theory editado por S. White. I. Shapiro se lamenta de que la disciplina de la $C P$ está demasiado method driven, en lugar de ser problem driven. 
Pero es también B. Parekh uno de los primeros en haberse «atrevido» a relativizar la proclamación del «declive», incluso «muerte», de la TP durante los años cincuenta y sesenta, en pleno período de «asalto» (en términos de A. W. Saxonhouse, 1993: 3) conductista y positivista. B. Parekh $(1968,2001)$ destaca rotundamente (él también era uno de los protagonistas) que durante los años cincuenta y sesenta la TP estaba viva, y no muerta, como se estaba abanderando desde el discurso hegemónico. Basándome en sus argumentos podemos destacar toda una serie de rasgos que caracterizan este período, cuya combinación provocó precisamente el hecho de que se dejase en segundo plano: ignorancia de los textos que se escribían (puesto que no seguían el orden establecido), menosprecio positivista, triunfalismo conductista, creencia en la inutilidad de mantener un diálogo con autores del pasado para resolver problemas presentes, creencia de que los problemas de los que se ocupaban eran caducos, contexto político adverso. Parekh subraya toda una serie de autores que, si bien no elaboraron grandes teorías del tipo Rousseau y Locke ${ }^{20}$, sí que contribuyeron a su desarrollo, sobre todo en un contexto intelectualmente adverso. Entre otros, destacan autores conocidos como I. Berlin, M. Oakeshott, L. Strauss, E. Voegelin, C. B. MacPherson, F. A. Hayeck, R. G. Collingwood, L. Althusser, Habermas, H. Arendt.

El artículo seminal de B. Parekh (1968) también debe ser interpretado en esta línea de apertura de la TP y de un «hacer frente» al conductismo reinante. Lo que quizás no fue consciente ni el mismo autor es que, al mismo tiempo que construía su argumentación en contra de los que proclamaban el final de la TP, estaba contribuyendo al revival de la disciplina. En este mismo momento, la TP comenzaba a «despertarse». Esto se producirá, como todos sabemos, y dos años más tarde, con la obra de J. Rawls (1971). Parekh interpreta que todas estas voces que proclaman la muerte de la TP no son más que síntomas de una crisis de identidad que se ha repetido en contextos históricos diferentes. En la fase histórica en la que se escribe el artículo, el autor insiste en que la TP no es sólo una tarea conceptual e historia del pensamiento político, sino también una reflexión sobre la actividad política y los principales factores de cambios. Dicha reflexión implica, a su vez, una exploración de las categorías, valores y principios implícitos en dicha actividad.

Lo cierto es que a mitad de los años sesenta el conductismo comienza a ser visto como lo que fue en realidad: una corriente que tiene en su misma base una lógica conservadora que sirvió para legitimar el orden político y social de posguerra. Será en el momento en que empieza a formarse una crítica contra este contexto que la TP se revitaliza. Las críticas fueron básicamente articuladas por académicos vinculados con la actividad teórica. Críticas contra el Vietnam, movimientos de derechos civiles, tensiones internacionales y Guerra Fría. Todos estos problemas no tenían un modo de articulación discursiva dentro de los 
parámetros fijados por el conductismo. En este contexto comienzan a aparecer importantes obras, como la de M. Walzer (1970) y la de C. B. MacPherson (1966, 1973), por citar a dos representativos. Pertenece igualmente a este período el artículo de Q. Skinner (1969), quien es considerado como el precursor de la reflexión metodológica en torno a la interpretación de textos en la Historia de la Teoría Política ${ }^{21}$. Desde el punto de vista de reconocimiento institucional de la disciplina, al final de esta etapa se deja en segundo plano, y por utilizar la distinción de J. C. Gunnell (1983), la concepción de la TP como subcampo de la $C P$, como discusión acerca del método. Se abre el período de la concepción de la TP como actividad intelectual independiente ${ }^{22}$.

\subsection{Consolidación 1970 - nuestros días}

Es ilustrativo que para caracterizar este período el mismo Gunnell (1983: 28) hable, ni más ni menos, de «diáspora de la $T P$ ». Visto desde los parámetros de la etapa anterior, se trata realmente de una «huida y éxodo» de la «jaula de hierro» conductista. Esto no significa que la TP como subcampo desaparezca, sino que permanecerá como TP empírica, pero ya no será el elemento exclusivo ni distintivo, como argumentaré en la sección 4. La TP será también considerada en su dimensión histórica, tratándose en este caso de un revival respecto a su etapa inicial. Pero lo nuevo será sobre todo su dimensión normativa. Si utilizamos como indicador el directorio de la APSA de 1968, la TP estaba oficialmente dividida en tres partes: histórica, normativa y empírica ${ }^{23}$. Utilizando la famosa imagen de G. Almond (1990), no será hasta este período cuando donde se institucionaliza un nueva «mesa» dentro de la variedad de «mesas separadas» que existen en la $C P^{24}$. Un indicador empírico que expresa este reconocimiento dentro de la $C P$ es la aparición de dos revistas especialmente centradas en la TP, y que desde entonces continúan siendo unas plataformas

21 En nuestro lenguaje, abre un programa de investigación que relaciona los Niveles de análisis 3 y 2. Una relación de la bibliografía básica está en nota 4.

22 J. C. Gunnell lo resume de la forma siguiente: «In the 1937 edition of the International Encyclopedia of the Social Sciences, not only was Political Theory not a separate heading, it was not even treated as a distinct sub-division in the discipline of political science. In the 1968 edition, Political Theory was not only given prominence and separate status in the section on Political Science, where it was treated as an activity, product, and sub-field, but it became a separate and equal topic where it was discussed almost as if it were an autonomous discipline with its various divisions, problems, dimensions, and history» (1983: 26).

23 Citado por Gunnell (1983: 19)

24 Aunque todavía sigue la confusión sobre si la TP debe ser considerada como una mesa distintiva o bien la propia «cafetería central» (y, por lo tanto, la que proporciona instrumentos, conceptos y elaboraciones teóricas a los demás campos). La TP como núcleo de unión de todas las ciencias políticas es percibida en su dimensión histórica (en el Nivel 2). Ésta es la línea que sigue, por ejemplo, G. Almond (VV.AA.,1990), quien parece inclinarse por contestar a la pregunta ¿cuál es el núcleo de la $C P$ ?: la Historia de la Teoría Política. Sería algo así como el café del centro, la central eléctrica de la $C P$. Sin $T P$ no es posible $C P$. La TP es la que proporciona unidad a la $C P$. 
importantes para conocer los debates y programas de investigación actuales. En 1971 aparece Philosophy and Public Affairs y, dos años más tarde, en 1973, la conocida Political Theory ${ }^{25}$. Un último elemento que acaba por configurar el inicio de este período de consolidación es, evidentemente, la aparición de A Theory of Justice (1971), de J. Rawls, verdadera «central eléctrica» de la TP durante los ochenta y noventa.

Las líneas básicas que caracterizan el debate de la TP en los inicios de este período son muy variadas, pero todas tienen una relación con el contexto de crisis generalizado y de cambio social (crisis de Estado, de Estado de Bienestar, fiscal, de legitimidad, de gobernabilidad, fiscal, etc.) y las respuestas políticas (neoconservadurismo de los años ochenta en Estados Unidos y en Gran Bretaña, por ejemplo) y sociales (nuevos movimientos sociales) que se están dando. Aunque en las secciones siguientes tendré espacio para detallar los principales programas de investigación y debates que se abren en este período, destacaré al menos tres grandes ejes que vertebran el universo discursivo de la TP: debate en torno a la Justicia y la distribución igualitaria de recursos, debate en torno a los «temas pendientes» de la democracia liberal y debate en torno a la democracia participativa ${ }^{26}$. Argumentaré que estos tres ejes quedan englobados en el debate más amplio en torno a la Ciudadanía Liberal Democrática.

En primer lugar, el discurso normativo en torno a la Justicia y a la distribución igualitaria de recursos, y todos sus debates colaterales (neutralidad, utilitarismo, por ejemplo) entre liberales (J. Rawls, 1971, 1993), comunitaristas (A. Maclntyre, 1981; M. Sandel, 1982), libertarios (R. Nozick, 1974), igualitaristas (R. Dworkin, 1977), republicanos (M. Walzer, 1983; Ch. Taylor, 1985, 1989), perfeccionistas (J. Raz, 1986; W. Galston, 1980), pragmatistas (R. Rorty, 1989, 1991), posmodernistas y teóricos críticos (J. Habermas, 1989; McCarthy, 1978; S. White, 1988) 27.

En segundo lugar, el debate en torno a los «temas pendientes de resolver» del sistema liberal democrático, el cual está muy conectado con la aparición y reivindicaciones de los nuevos movimientos sociales. Entre otras, destacan las teorías feministas y teorías ecologistas (también llamada teoría política verde). En general, estos temas se centran en la configuración y gestión del espacio público, en el reconocimiento de la identidad política. En este marco, también podemos destacar una revisión de los conceptos claves que articulan el debate, como los de autonomía, representación, ciudadanía, poder. Se abre inclu-

\footnotetext{
25 Hacer un análisis de sus índices es repasar la reflexión de la TP desde los setenta. Un estudio que queda todavía por hacer.

26 Las dos siguientes secciones, aunque separadas, pueden ser consideradas como continuación de la breve descripción que estoy haciendo de este período.

27 Un manual de referencia que retrata los debates de los años setenta y ochenta sigue siendo S. Mulhall y A. Swift (1992), W. Kymlicka (1989, 1990).
} 
so una colección de la editorial Open University Press centrada precisamente en los conceptos básicos de las ciencias sociales. Entre los más destacados y citados se encuentran análisis de conceptos fundamentales como el de Democracia (A. Arblaster, 1992), Ciudadanía (J. M. Barbalet, 1988), Liberalismo (J. Gray, 1994), hasta llegar al reciente análisis de Multiculturalismo (C. W. Watson, 2000). En esta línea, también destaca la serie de temas de Teoría Política de la editorial MacMillan, dirigida por P. Jones y A. Weale. Entre otros temas analizados, han aparecido estudios interesantes en torno a la Autonomía (R. Lindley, 1986), Legitimidad (D. Beetham, 1991), Tolerancia (S. Mendus, 1989).

En tercer lugar, todo un debate en torno a la democracia y las teorías participacionistas, y el papel que debe jugar la sociedad civil frente al conservadurismo reinante. En este marco aparecen temas en la agenda relacionados, entre los que destaca una reflexión sobre qué significa «representar» cuando lo utilizamos para calificar el tipo de democracia que tenemos. Entre las propuestas, se produjo todo un debate en torno al uso de los medios tecnológicos para reducir la distancia que existe entre la ciudadanía y sus representantes, permitiendo a los primeros participar directamente en la toma de decisiones políticas ${ }^{28}$. Recientemente, y siguiendo esta línea de propuestas para colmar las deficiencias de la democracia representativa, han aparecido líneas en torno a la democracia deliberativa, muy conectado con el recientemente revitalizado enfoque republicano (Ph. Pettit, 1999).

La lógica básica se concentra en criticar el enfoque citizen/voter y defender una perspectiva más centrada en el citizen/politician. Aunque el lenguaje en un principio era alternativo, hoy en día podemos decir que es correctivo de la democracia liberal representativa. Los principales teóricos participacionistas o defensores de la democracia directa tienen, además, un contexto de aplicación en el trasfondo de sus razonamientos. Entre los más influyentes destacan C. B. MacPherson (1966, 1977), en un nivel de aplicación general; C. Pateman (1970), más centrado en el nivel laboral, y B. Barber (1984), fundamentalmente dirigido hacia el nivel local y vecinal.

Si me preguntasen cuál es el elemento catalizador de estos tres ejes, respondería que es el debate en torno a la ciudadanía democrática liberal. Este tema ha sido el principal motor de los debates de los años ochenta y noventa, y ha actuado como principal variable explicativa.

No será hasta entrados en los años noventa (después de los sucesos del 89) y el final de la Guerra Fría cuando se incorpora de forma directa un nuevo tema en la agenda: el del pluralismo cultural o multiculturalismo y su impacto sobre los principios legitimadores de la democracia y del liberalismo (R. Zapata, 2001b). Este nuevo programa de investigación se in-

28 Al respecto, véanse, entre los primeros defensores, a C. B. MacPherson (1966), R. P. Wolff (1970: 34-37), B. Barber (1984: 273-278, 289-290), R. A. Dahl (1989), I. Budge (1993). 
corpora en cada uno de los tres ejes anteriores, jugando el papel de perspectiva dominante similar al debate en torno a la ciudadanía. Esto explicaría la resonancia que han tenido y siguen jugando (aunque con menor intensidad) los trabajos de W. Kymlicka (1995) en torno a una ciudadanía multicultural. Kymlicka ha sabido vincular precisamente los dos principales motores del debate de la TP de los ochenta y noventa, convirtiendo dicho vínculo en nuevo programa de investigación.

Si bien tenemos a la ciudadanía y al multiculturalismo como las dos perspectivas analíticas dominantes, existe un telón de fondo supuesto que precisamente la TP de principios del 2000 debería comenzar a discutir. B. Parekh (2001) es quizás uno de los que constantemente ha dedicado una parte de su trayectoria intelectual a subrayar estos supuestos del debate, que ni tan siquiera M. Walzer, uno de los que también se ha dedicado a esta tarea crítica, ha sabido detectar con tanta claridad. Podríamos destacar dos rasgos mayores de la reciente TP:

- Triunfalismo del liberalismo o el liberalismo como nueva jaula de hierro, hegemónico en el sentido gramsciano, como patrón absoluto de medida para la evaluación. En el lenguaje que he estado utilizando anteriormente, podríamos decir que el liberalismo actúa como esfera dominante, discurso hegemónico, puesto que es el contexto, el objeto y el marco de referencia de la TP. Esta omnipresencia es tal que no es posible hacer TP fuera de dicho «círculo de comprensión», por utilizar una expresión gadameriana. Parekh dice que el liberalismo se ha convertido en un metalenguaje que es al mismo tiempo lenguaje como los demás, árbitro de cómo los demás lenguajes deben hablarse, es decir, una especie de medida que es la medida de todas las monedas. En términos económicos, diríamos que el liberalismo se ha convertido en el patrón oro. Por utilizar una expresión de Gunnell (1986), el liberalismo es el principal elemento que aliena el debate de la TP.

- Etnocentrismo. Es una TP muy etnocéntrica, «provinciana», en tanto que ha desestimado a «los otros» no occidentales. Los debates tienden a llevarse a cabo con unos supuestos de valores muy enraizados a nuestra tradición ilustrada, tendente a percibir la heterogeneidad y diversidad en términos «anómalos» y conflictivos.

En este marco, en estos primeros años del 2000, si bien la ciudadanía y el multiculturalismo continúan siendo las perspectivas analíticas dominantes, el tipo de análisis que se están produciendo tienden a escapar del método deductivista y universalista predominante y a contemplar la inferencia como uno de los recursos metodológicos más adecuados para conducir los argumentos y construir teorías. Este recurso práctico a la inferencia tiene dos ejes principales: el enfoque pluralista y el enfoque contextualista. Ambos están aportando (y aportarán en un futuro próximo) nuevas líneas de investigación dentro de los programas dominantes. El enfoque pluralista, representado por el reciente libro de B. Parekh (2000), cuestiona precisamente el argumento que la homogeneidad es lo normal y la heterogeneidad es algo anormal, muy 
consolidado en muchos discursos de nuestros políticos; el enfoque contextualista, potenciado por el interesante estudio de J. Carens (2000), incide, además, en que no se puede argumentar sin tener en cuenta el marco contextual. El contexto es el que dota de sentido a los principales conceptos y argumentos. Los límites de este contexto los debe poner el propio teórico político. Juega el papel de marco de referencia y fuente generativa constante de significados. Este contexto puede ser un territorio, un período histórico, una situación concreta. Estos dos enfoques, el pluralista y el contextualista, darán mucho que hablar en el futuro inmediato ${ }^{29}$.

\section{PRINCIPALES PROGRAMAS DE INVESTIGACIÓN QUE CONTRIBUYEN AL ESTUDIO DE LOS PROCESOS DE CAMBIOS ESTRUCTURALES}

Destacar los principales programas de investigación de la TP es una empresa difícil; pretender que dicha clasificación sea correcta y exhaustiva es pretencioso; aspirar a destacar sus principales representantes es arriesgado. Lo único seguro es que, cualquiera que sea la tipología que hagamos, se confirmará el argumento principal: la TP ofrece elementos para orientar y dar sentido a los procesos de cambios sociales y políticos que vivimos. El mayor problema estriba en la dificultad de encontrar un marco de referencia con un hilo conductor coherente que pueda englobar a todas las líneas de investigación. Es inevitable que se produzcan solapamientos entre ellos y confusión constante entre categorías y subcategorías, entre contenido y continente. Lo que aquí enseñaré serán, pues, unos «apuntes» ilustrativos. Seguramente, no estarán todos los autores, pero creo que puedo afirmar que todos los que he incluido son importantes si uno, desde fuera, quiere comenzar a entrar en un programa de investigación determinado.

Lo que sí está claro es que cualquier clasificación que hagamos tendrá dos características. Por un lado, debe mostrar la diversidad de temas que existen, teniendo muy en cuenta que dicha diversidad no implica dispersión. Si dejamos de lado nuestra mente ilustrada, debemos concebir la diversidad de programas como signo de buena salud de una disciplina, puesto que expresa constante movimiento de autores y temas, provocando renovaciones de programas de investigación y vitalidad innovativa.

Por otro lado, y quizás más importante, puesto que se alinea a la concepción de la TP que estamos respaldando, la clasificación que se haga deberá coincidir con los principales temas de la agenda política y social. Hasta tal punto esta conexión es necesaria que puede muy bien servir de argumento para decir que alguien está trabajando sobre temas caducos o anacrónicos el hecho de que su programa de investigación no coincida con la agenda social y política.

\footnotetext{
29 Siguiendo esta línea de análisis, un libro reciente cuestiona precisamente el enfoque «norteamericano» del multiculturalis-
} mo frente a un enfoque europeo. Véase T. Modood, A. Triandafyllidou y R. Zapata Barrero (2005). 
Para llevar a cabo esta clasificación me he planteado la siguiente situación. En un primer encuentro entre dos colegas, descubren que hacen TP y se preguntan en qué área o tema trabajan. En la primera columna de la tabla siguiente aparecen las posibles respuestas que podrían darse. Asimismo, una vez resuelto el tema del área, la siguiente pregunta que se plantearían estos dos supuestos colegas es sobre los autores que precisamente han abierto y están contribuyendo a la consolidación de dicha área como programa de investigación. Éste es el sentido de la segunda columna ${ }^{30}$.

Programas de investigación

Justicia social y teoría de los derechos (distribución de bienes y desigualdades)

Teorías de la democracia (enfoques participativos, representación, deliberativos, asociativos)

Feminismo

Posmodernidad

Demandas de grupo

Sociedad civil

Sociedad civil

Política verde

Estados de Bienestar y Políticas públicas

Relaciones internacionales, globalización, cosmopolitismo, Teoría Política Comparativa

B. Barry y R. Goodin (eds. 1992), J. Carens (2000), R. Bauböck et al. (eds., Inmigración, Multiculturalidad
A. Arato y J. Cohen (1992), J. Keane (1988), J. Cohen (1983)

R. E. Goodin (1992), J. Dryzek (1987), B. Duherty y M. De Geus (eds. 1996), A. Dobson (1997)

P. Self (1988), R. Goodin (1982), N. Luhman (1993)

Ch. Beitz (1979), R. B. Walker (1993), D. Held (1997), A. J. Parel y R. C. Keith (eds., 1992), F. Dallmayr (ed. 1999), N. O'Sullivan (2000)

J. Rawls (1971, 1993), R. Nozick (1974), B. Barry (1989), B. Ackerman (1980), A. Sen (1992), M. Walzer (1983), R. Goodin (1988), D. Miller (1976)

C. Pateman (1970), C. B. MacPherson (1973, 1987), M. Walzer (1983), J. Mansbridge (1980), B. Barber (1984), J. Drysek (1990), J. S. Fishkin (1991), P. Hirst (1994), N. Bobbio (1984), J. Burnheim (1985), R. Dahl (1989), Ch. Mouffe (ed, 1992), A. Phillips (1991), M. Freedem (1996)

S. M. Okin (1989), L. Clark y L. Lange (1979), J. B. Elshtain (1981), L. Ni(1985)

A. Honneth (1991), S. White (1991), K. von Beyne (1994), B. Honig (1993), R. Rorty (1989)

W. Kymlicka (1989), Ch. Taylor (1992), I. M. Young (2000), D. Miller (1995) 1996), B. Parekh (2000), T. Hammar (1990), W. Schwartz (ed., 1995), V. Bader (ed., 1997), R. Brubaker (ed., 1989) cholson (1986), I. M. Young (1990), M. L. Shanley y C. Pateman (eds.

30 Para elaborar este cuadro he utilizado una amplia literatura. Distinguiría principalmente los siguientes: C. J. Nederman y P. M. Elliot (1983), Galston (1993), I. M. Young (2001), B. Parekh (2001). Destacamos, igualmente, que existen autores que aparecen en más de un programa en tanto que sus líneas de trabajo varían a lo largo del tiempo o pueden clasificarse en más de un programa. Indico, además, autores de libros y no de artículos, para no alargar demasiado la lista. Aparecen algunos con tan sólo artículos, pero sólo en ocasiones en donde lo he considerado imprescindible. 
Un análisis minucioso de los temas que se debaten en estos programas de investigación, las fracturas y polarizaciones que existen en sus discusiones, rebasaría el marco de este artículo (es otro tema pendiente de hacer). Lo que aquí nos interesa subrayar es que si tomamos los programas de investigación anteriores en cuenta, constatamos que es una mezcla de muchas perspectivas. Aunque es muy difícil determinar un único criterio que los incluya a todos, sí que podemos considerar al menos los tres ejes anteriores que hemos dado, además de otros también importantes. Utilizando la imagen de G. Almond, si consideramos que cada programa de investigación es una mesa donde se "cocina» y se discute durante la comida, la "cafetería central», considerada como el espacio de encuentro de todos, debería contener los ejes en torno a la ciudadanía, el multiculturalismo, el liberalismo y la democracia.

\section{PRINCIPALES ENFOQUES: CONCEPCIÓN FUERTE Y DÉBIL DE LA TP}

Adoptando una perspectiva analítica, la literatura existente destaca al menos tres formas diferentes de llevar a cabo la $T P^{31}$. Puede seguir un enfoque empírico, formal y normativo. A continuación me ocuparé de describir brevemente cada uno de estos enfoques, pero defendiendo un argumento. Diferenciaré la TP en sentido débil y la TP en sentido fuerte. En el primer sentido incluiré el enfoque empírico y formal; en la segunda forma englobaré el enfoque normativo e institucional. Mantendré que la concepción que proporciona más sentido a la tarea de la TP como gestora de cambios y con potencial innovador es la concepción fuerte, correspondiente a una concepción de la TP como una actividad con fines propios. Los otros dos enfoques correspondientes al sentido débil consideran la TP como una actividad instrumental para llevar a cabo objetivos de investigación pertenecientes a otros campos de la $C P$, como una subdisciplina supeditada a una disciplina mayor.

\subsection{La Teoría Política en sentido débil: enfoque empírico y enfoque formal}

La TP empírica es una reflexión acerca del método y de los resultados de las investigaciones realizadas en los otros campos de la CP. Como reflexión metodológica, corresponde estrictamente a las primeras partes de las investigaciones empíricas. Son literalmente las partes metodológicas de la $C P$. El vínculo entre teoría y metodología es tan estrecho que generalmente se toma la una por la otra. Aunque en menor medida, todavía existen revis-

31 Me baso, entre otros, en D. Miller (con L. Siedentop, eds.,1983; 1987, 1990), Ph. Pettit (1991), D. Held (1991), R. E. Goodin y Ph. Pettit (eds., 1993), R. Bellamy (1993), B. Barry (2001), K. von Beyne (2001). 
tas y manuales de $C P$ que incluyen la $T P$ en la misma sección que la metodología ${ }^{32}$. Incluso esta parte metodológica se llega a vincular a la reflexión de la CP como disciplina ${ }^{33}$. Como «partes teóricas» de una investigación empírica, la TP se ocuparía de diseñar y aplicar las correlaciones y factores explicativos, utilizando las dicotomías entre variables dependientes y variables independientes, entre explanans y explanandum.

Desde este punto de vista instrumental, la TP se asocia también con los resultados de una investigación empírica. En este marco existen grandes teorías empíricas, como el consocialismo (A. Lijphart, 1977, 1984), el neocorporatismo (Ph. Schmitter y G. Lehmbruch, eds., 1979), las teorías de la democratización y de las transiciones a la democracia, desarrolladas siguiendo los procesos reales que se estaban produciendo en el sur de Europa y América Latina (Ph. Schmitter, G. O'Donnell y L. Whitehead, 1986; J. Linz y A. Stepan, 1978); y, más recientemente, teorías de la sociedad del riesgo (U. Beck, 1992), las teorías de los «tres mundos de Estados de Bienestar» de G. Esping-Andersen (1990), o la tan utilizada teoría del capital social de R. Putnam (1993).

En este marco, es útil la distinción que hace K. Beyme (2001: 749) entre dos corrientes principales dentro de esta segunda forma de concebir la TP empírica: la corriente weberiana, caracterizada por su perspectiva histórica y la construcción de tipologías ideales; y la durkheimiana, centrada sobre todo en la explicación causal, utilizando el recurso de variables dependientes e independientes.

La TP formal, por su parte, es una actividad que se identifica con la construcción de modelos. Se aplica tanto en el diseño de modelos empíricos como teóricos. En el primer caso, está conectada con la perspectiva de la elección social. Se trataría de diseñar modelos teniendo en cuenta ciertos actores, sus preferencias, ciertas finalidades y procedimientos y reglas. Destacarían las teorías con enfoque de la elección racional y teoría de juegos $^{34}$.

Esta tendencia a construir modelos también se aplicó en el análisis de conceptos específicos. Este intento de entender la teorización como construcción de modelos fue sobre todo

\footnotetext{
32 Véase, por ejemplo, el índice de A. Finifter (1983), quien titula la primera sección como «Theory and Method». También hemos constatado que en el apartado de recensiones de la APSJ juntan en una misma sección la TP y la metodología.

33 La segunda edición del manual de A. Finifter (1993), aunque mejora el contenido de su índice respecto de la primera edición (1983), introduce los capítulos sobre la TP en la sección dedicada a hacer un estado de la cuestión de la CP: «Political Science: the discipline and its scope and theory".

34 Véase la contribución de B. Barry (2001) en el manual de R. Goodin y H. Klingemann, especialmente su bibliografía introductoria, para seguir estos temas más formales.
} 
aplicado en el conocido trabajo sobre Modelos de democracia de D. Held (1987), así como modelos de burocracia o de legitimidad en los análisis conceptuales de D. Beetham (1987, 1991).

\subsection{La Teoría Política en sentido fuerte: enfoque normativo (basado en principios y en instituciones)}

La TP normativa se podría describir como gestión de desideratums de la sociedad. Se articula principalmente con valores, principios y criterios, y con las instituciones que los fomentan, los distribuyen y los protegen. Es la concepción de la TP reconocida por su carácter distintivo respecto a otros tipos de reflexiones teóricas. Éste es el enfoque consolidado a partir de los años setenta. Como ya he tenido ocasión de precisar su contenido en otros apartados, por tratarse del enfoque básico que estoy defendiendo, me ocuparé aquí de recoger los puntos más relevantes.

La TP en sentido fuerte se interesa principalmente por destacar las normas y los valores morales, políticos y culturales que ayudan a justificar los argumentos, las decisiones y las acciones que tienen un alcance político. Trata de reflexionar sobre los principios y los criterios que legitiman las acciones e instituciones políticas. Se sigue como primera constatación que se asume la indivisibilidad de hechos y valores. Por lo tanto, tiene una gramática y sigue una lógica totalmente opuesta al lenguaje conductista de los años cincuenta y sesenta. Una de sus tareas es, precisamente, inferir de los hechos todos los valores que llevan implícitos. Este recurso metodológico a la inferencia es una de sus características diferenciadoras. De cada hecho, acción, institución, trata de extraer un sistema de justificaciones. Este sistema puede ser utilizado de forma descriptiva y/o explicativa, pero fundamentalmente se usa de forma prescriptiva y evaluativa. Esto explica que la TP normativa tenga su máxima expresión cuando entra en el terreno del deber ser, de lo deseable (por ejemplo, Ph. Pettit, 1991).

Si profundizamos este enfoque, toda teoría normativa descansa sobre una concepción de la persona (antropología) determinada, y a partir de dicha base trata de proponer instituciones que sean congruentes. De hecho, la relación entre concepción antropológica y la propuesta de arreglos institucionales es, quizás, uno de sus epicentros. En este aspecto, la teoría normativa se rige por el principio de la congruencia entre la concepción de la persona y el modo en que se articulan la sociedad y la política. Al reflexionar sobre procedimientos e instituciones, se interesa sobre todo por analizar los principios (de justicia, de igualdad, de libertad, de las necesidades humanas, de los derechos humanos, etc.) que justifican su existencia y que legitiman su actividad. Desde este punto de vista, la TP en sentido fuerte se ocupa básicamente de los criterios de legitimación de las instituciones. 
Quizás las palabras de J. Plamenatz son las que resumen mejor este enfoque normativo:

«By political theory I do not mean explanations of how governments function; I mean systematic thinking about the purposes of government» (1960: 37).

Esto significa que el centro de atención de la TP en sentido fuerte es eminentemente finalista y centrado en los valores últimos que legitiman toda actividad política. Su tarea es, pues, evaluativa y no estrictamente explicativa. De hecho, la teoría política explicativa pertenecería más bien a la que orienta los estudios empíricos (en sentido débil, tal como lo hemos categorizado aquí). Como sabemos, J. Rawls (1971) es uno de los primeros en iniciar este tipo de análisis y en haber abierto un importante debate acerca de la compatibilidad entre los principios de la libertad y de la igualdad, de la justicia y de la imparcialidad en las decisiones políticas, etc. Todos estos temas son los que conforman hoy en día el debate normativo.

Siguiendo esta orientación normativa, podemos dividir el enfoque normativo en una reflexión sobre principios (libertad, igualdad, derechos, etc.) y los arreglos institucionales que se utilizan para llevar a cabo estos principios. En este caso, el enfoque normativo no está basado en principios, sino en instituciones. En este sentido, la TP normativa también es una reflexión institucional. Se ocupa de los hechos y del ser. Trata las instituciones como variables dependientes. Desde la perspectiva de las instituciones se ocupa de inferir los principios generales que justifican la existencia misma de dichos hechos e instituciones, de sus actividades a través de decisiones y políticas públicas. Se interesa también por destacar los conceptos claves y los principios que legitiman las instituciones y guían sus actividades. En este enfoque incluiría, por lo tanto, tanto el análisis legitimador de las instituciones como el análisis teórico de las políticas públicas (R. Goodin, 1982; D. Miller, 1990). Aquí entrarían, por ejemplo, los estudios normativos institucionalistas promovidos por, entre otros, A. Hamlin y Ph. Pettit (eds., 1989).

Estos enfoques de la TP constituyen una división real de trabajo dentro de los departamentos, aunque en la práctica investigadora dicha separación es más difícil de perfilar, puesto que cada tipo de TP se relaciona con los otros en alguna fase de la investigación (D. Miller, 1987: xxii-xxiv). Pienso que lo más interesante de la actividad de la TP es precisamente saber combinar estas perspectivas. Diría incluso que es esta combinación la que dota a la TP de una distinción y autonomía. En este sentido, si tomamos el enfoque de la TP en sentido fuerte, lo que dota de sentido normativo a la misma actividad teórica es el ir y venir de lo institucional a los principios, del ser al deber ser, de los hechos a los valores. 
Como podemos inferir hasta el momento, la TP es principalmente una tarea analítica y conceptual vinculada directamente con la realidad política y social ${ }^{35}$. Trata de los «asuntos complejos", de los temas prioritarios de la agenda política y social, intentando separar en teoría los elementos que aparecen vinculados o mezclados en la práctica. Su principal tarea interpretativa es, pues, intentar dar sentido y claridad sobre acontecimientos o hechos prácticos que confunden y desorientan. Para llevar a cabo su actividad utiliza, como hemos visto, argumentos, valores, principios, fundamentos, conceptos, y los aplica a la realidad que quiere analizar en un segundo orden, ocupándose de aquello que se da por supuesto, de las ideas recibidas, del sistema de creencias o los «mitos». En este sentido, su tarea podría describirse como desacralizadora, desencantadora en términos weberianos. Junto con esta actividad hermenéutica, su función también es prescriptiva, en el sentido de que da por supuesto que es posible pasar del ser al deber ser. El deber ser, en este caso, le permite formular críticas al ser y darle recomendaciones. El teórico político debe saber moverse con facilidad entre el ser y el deber ser. Podemos decir que el ser limita lo que debe ser, y el deber ser le permite criticar y modificar el ser. Sin este movimiento dialéctico, la TP perdería gran parte de su potencial «generador de innovación».

\section{TEORÍA POLÍTICA Y ÁREAS DE INNOVACIÓN POLÍTICA}

¿Cuáles son los principales retos de la TP hoy en día? ¿Cuáles son sus principales áreas de innovación? Podemos entender esta pregunta de dos maneras. O bien considerando los temas actuales de la agenda de la TP, o bien los retos que presenta como disciplina. Consideraré los dos sentidos respectivamente, aunque ampliaré más el segundo, del cual he hablado menos en las secciones anteriores.

Probablemente, si hiciéramos como ejercicio anotar en una hoja los temas que ocupan la TP hoy en día, destacando los problemas actuales con los que se deben enfrentar la sociedad y la política, llegaríamos a la siguiente conclusión. Casi todos los temas básicos de nuestra época tienen que ver con el multiculturalismo en todas sus vertientes y la justicia distributiva. Probablemente también, observaríamos que la mayoría de los problemas no pueden estar en manos de unos Estados, sino que debe ser resultado de una interrelación entre Estados. Éstos son los temas de los que se ocupa la TP, de los problemas y conflictos que ponen en duda nuestros cimientos liberales y democráticos, los que afectan directamente la estabilidad de la sociedad y ponen en entredicho la capacidad de gestionar estos nuevos asuntos. En resumidas cuentas, uno de los mayores desafíos de la TP

35 Ésta es la principal perspectiva que proporcionan D. Miller y L. Siedentop (1983), como actividad académica distintiva. 
es el de saber identificar y denunciar las contradicciones existentes entre los valores democráticos y liberales, fundamentados en los derechos humanos, y las prácticas de los Estados que constantemente los vulneran en nombre precisamente de dichos valores. En este marco, cada vez se perfila mejor que la función de la TP es la de destacar la producción de paradojas (S. White, 2002: 474), contradicciones, incoherencias e inconmensurabilidades (J. Raz, 1986; Ch. Taylor; T. 1985; T. S. Kuhn, 1962, 2000) entre la práctica y los principios.

Así pues, y siguiendo el primer sentido de la pregunta que he planteado, diría, en congruencia con la concepción que estoy defendiendo, que los retos de la TP son los retos de la sociedad política hoy en día. Pero considerando la pregunta en su segundo sentido, nos referimos a la TP como disciplina. En esta línea, destacaría muy brevemente tres tipos de desafíos: i) profundizar programas de investigación ya abiertos; ii) abrir nuevas líneas de investigación, y iii) profundizar los aspectos metodológicos.

i) Como ya he señalado, la mayoría de los programas de investigación actuales se basan en el triángulo siguiente, considerado como contexto analítico dominante: Ciudadanía/Multiculturalismo/Democracia liberal. Cada uno de los componentes de esta relación debe considerarse en todas sus dimensiones y niveles de análisis. En este marco, y en general, la mayoría de la producción teórica formula argumentos para consolidar y/o criticar el paradigma moderno centrado en el vínculo indivisible entre el Estado, la Nación y la Ciudadanía (R. Zapata, coord., 2001a; 2002; 2004a). Las discusiones comienzan ahora a considerar la diversidad y el pluralismo culturales como la norma y no la excepción (aunque todavía existe una enorme tarea por hacer) ${ }^{36}$. La mayoría de la producción teórica había supuesto la necesidad de que la sociedad sea homogénea para justificar y apoyar las estructuras políticas. Hoy en día, este supuesto está siendo directamente objeto profundo de revisión. Tras los sucesos del 11 de septiembre de 2001 y el auge de los populismos con discursos concentrados en el vínculo de la inmigración y la inseguridad, existen algunas voces que apuntan al final de la Era del Multiculturalismo (los años noventa) y que ahora se abre una nueva fase «conservadora»37.

ii) Las nuevas líneas de investigación consolidarán precisamente estos debates, aclarando analíticamente algunas confusiones actuales, como, por ejemplo, el implícito entre gestión del pluralismo cultural y gestión del pluralismo religioso. Se abrirán también

36 En este marco, los diferentes trabajos de F. Requejo son un material sugerente. Véanse, entre otros, F. Requejo (ed., 2001; 2005) y R. Máiz y F. Requejo (eds., 2005).

37 Véase el artículo de J. Lloyd (2002). Se trata este tema en R. Zapata Barrero (2004a). 
nuevas líneas conectando dimensiones existentes en cada uno de los componentes. Por ejemplo, la conexión entre multinacionalidad/inmigración/democracia, Derechos Humanos/Liberalismo/Seguridad. En este sentido, y en términos de M. Dogan (2001) cuando analiza las áreas y subáreas de la $C P$, seguramente que las nuevas líneas de investigación que se abran serán híbridas.

iii) Desde el punto de vista de «innovación» metodológica, se reforzarán más las reflexiones que utilicen el recurso a la inferencia, intentando partir de contextos concretos y extraer de ellos el máximo de categorías y de información bajo forma de principios, conceptos y valores, con el fin de proceder a la reflexión normativa e institucional. En este marco, las perspectivas pluralistas (B. Parekh, 2000) y contextualistas (J. Carens, 2000) acaban justo de comenzar a dar sus primeros resulta$\operatorname{dos}^{38}$. Ambos nuevos enfoques compartirían la convicción rortyana de que no es posible encontrar un «punto de Arquímedes» que nos permita estar fuera de los contextos específicos y evaluar al mismo tiempo los sistemas políticos o mediar entre valores conflictivos (R. Rorty, 1989; R. Bellamy, 1993: 11).

Comparto asimismo el diagnóstico que efectúa S. White en el número de agosto de 2002 de Political Theory, quien no sólo nos recuerda los retos actuales como el feminismo, medio ambiente, teoría de la raza (diría mejor «inmigración»; me parece todavía increíble que la palabra «raza» no haya desaparecido del vocabulario político a estas primeras alturas del siglo XXI), nacionalismo, cosmopolitismo (2002: 474), sino también los siguientes temas urgentes: el compromiso liberal con el pluralismo, teniendo en cuenta sus nuevas formas; la penetración planetaria del capitalismo que genera como reacción movimientos anti-globalización; las dificultades de la democracia y de crear espacios públicos democráticos (2002: 475-476). A esta lista añadiría también una cuarta señalada por B. Parekh en reiteradas ocasiones (por ejemplo, 2001: 742-746). Intentar construir en serio un lenguaje alternativo. Este discurso se cimentaría no solamente contra al universalismo reinante (especialmente porque supone una homogeneidad cultural en su aplicación planetaria o en contextos de diversidad cultural), sino también, y en conexión con el anterior, contra toda forma monocultural de concebir el mundo.

\section{REFLEXIONES FINALES}

La TP tiene algo de contra-corriente, en el sentido que su vitalidad y expansión se producen en tiempos de crisis y de conflictos. Constatamos, por ejemplo, que la periodización

\footnotetext{
38 Discuto todo esto en el último capítulo de R. Zapata Barrero (2004a).
} 
que he efectuado de las dos últimas etapas, la etapa de la «incertidumbre (1945-1970)» y el período actual de «consolidación (1970 - nuestros días)», es contraria a la que hubiéramos hecho si tratásemos de los Estados de Bienestar o de otras disciplinas. Intentaré abordar esta última sección siguiendo precisamente este hilo conductor.

La TP no tiene sólo el conflicto como principal objeto de estudio, sino que su área de investigación siempre se sitúa en los límites del sistema liberal democrático. Hasta tal punto es esto cierto que podemos aseverar que la TP es una reflexión permanente sobre la teoría liberal y democrática, nuestra propia consciencia como sociedad y forma de organizarnos políticamente. Son esos conflictos fuertes los que le interesan particularmente, y no tanto los débiles o «cotidianos» que puede resolver el propio sistema sin poner en duda su consistencia y coherencia, su propia estabilidad y permanencia a lo largo del tiempo. En este sentido, la tarea propia de la TP es analizar los elementos motores de la sociedad, de cambios de paradigma. No consiste sólo en identificar este tipo de conflictos fuertes, sino que expresa también la consciencia de que en la resolución de dichos conflictos se deberán producir cambios estructurales, transformaciones sociales y políticas.

Se interesa, pues, por describir y analizar los cambios revolucionarios y no sólo normales, en el sentido fuerte que le daba Kuhn. Es decir, aquellos aspectos que implican cambios de parámetros, de conceptos y nociones, ya que los viejos parámetros no son útiles para explicar la nueva realidad. Genera argumentos a partir de la tensión entre recursos conceptuales tradicionales y problemas nuevos. De ahí que se vayan abriendo tantos programas de investigación como elementos de cambios estructurales. En términos de Pocock, también podemos decir que la TP intenta localizar los elementos que configuran los «momentos» históricos. Lo interesante del momento actual es que pone signos de interrogación sobre hechos, conceptos y creencias que dábamos por supuestos tan sólo hace unos años, plantea preguntas que generaciones anteriores ni tan siquiera hubieran podido imaginar. Saber captar estos «momentos» es una de sus posiciones. En esta línea, podemos decir que la innovación en la TP hoy en día puede tener una doble vía: plantearse preguntas nuevas, o plantearse preguntas tradicionales pero ofreciendo nuevas respuestas.

Desde un punto de vista contextual, es también materia de reflexión el hecho de que el debate de la TP se europeíce en el doble sentido. Es decir, que el lenguaje sea más europeo y que los temas sean aquellos que más directamente afecten a Europa. Se debe intentar reflexionar, pues, sobre la necesidad de construir no sólo una TP Europea, sino también una TP sobre Europa. Es una realidad que la TP, como tantas otras áreas de la $C P$, está demasiado dominada por la academia norteamericana. Este hecho, en sí, no es negativo. 
Pero sí que se convierten al menos en materia de reflexión las confusiones que se producen en el lenguaje precisamente porque se intentan aplicar resultados de las reflexiones hechas en un marco contextual norteamericano en Europa. Europeizar el discurso de la TP es un tema de debate pendiente para el futuro ${ }^{39}$.

\section{APÉNDICE. LA TEORÍA POLÍTICA EN ESPAÑA:}

\section{APUNTES PARA ABRIR UN PROGRAMA DE INVESTIGACIÓN}

Una reflexión sobre la TP en España es un asunto pendiente. Quizás sea todavía demasiado prematuro emprender un estudio de estas características, por ser una tarea académica demasiado joven. Pero creo que es, sin duda, un ejercicio práctico comenzar ya a trazar las primeras líneas de un programa futuro de investigación. Éste es el principal objetivo de esta sección. Intentaré plantearme la situación en que un académico extranjero me formule la pregunta: ¿desde cuándo se practica y de qué se ocupa la TP en España?

Para empezar, utilizaría una serie de indicadores para destacar su reconocimiento institucional como tarea académica dentro de la CP. El primer indicador temporal sería, sin duda, el de la aparición de la Historia de la Teoría Política de F. Vallespín (1990), pero también la organización de mesas de trabajo en el área temática de Teoría Política desde el I Congreso de Ciencia Política y de la Administración (Bilbao, 1994), precisamente coordinado por F. Vallespín. También mencionaría la aparición de Centros de estudios especialmente dedicados a la TP dentro de las licenciaturas de CP existentes en España, especialmente el Centro de Teoría Política de la UAM (creado en 1991; véase http://www.uam.es/ otroscentros/teoriapolitica/default.html [junio 2005]) y el más reciente Grup de Recerca de Teoria Política (GRTP) de la UPF (www.upf.edu/dcpis/grtp). Asimismo, destacaría la producción de libros relacionados con la materia desde los volúmenes de la Historia de F. VaIlespín hasta el monográfico especial de la reciente Revista Española de Ciencia Política (n. ${ }^{\circ} 3,2000$ ). Para contestar a la pregunta acerca de los temas de los que se ocupa detallaré el contenido de algunos indicadores institucionales. Comencemos por los temas abordados en los diferentes Congresos de $C P$.

\footnotetext{
39 En esta línea, por ejemplo, acaba de aparecer la nueva revista European Journal of Political Theory (2002), con el objetivo
} de complementar la ya histórica Political Theory americana. 
Congresos

Grupos de trabajo

\begin{tabular}{|c|c|}
\hline Bilbao, 1994 & $\begin{array}{l}\text { - Legitimación política en las democracias liberales } \\
\text { - La construcción de las identidades políticas } \\
\text { - Las escisiones de la democracia } \\
\text { - Teoría Política en España hoy }\end{array}$ \\
\hline $\begin{array}{l}\text { Santiago de } \\
\text { Compostela, } 1996\end{array}$ & $\begin{array}{l}\text { - Ideologías políticas contemporáneas } \\
\text { - Multiculturalismo, nacionalismo y federalismo. La revisión del liberalismo en los años noventa } \\
\text { - Teorías de la democracia } \\
\text { - Ideas Políticas y de Estado }\end{array}$ \\
\hline Salamanca, 1997 & $\begin{array}{l}\text { - Lemocracia y sociedad civil } \\
\text { mo y nacionalismo } \\
\text { - La teoría y la práctica de la ciudadanía: problemas de definición y problemas de inclusión } \\
\text { - Economía Política } \\
\text { - Pensamiento político en el cambio de siglo }\end{array}$ \\
\hline Granada, 1999 & $\begin{array}{l}\text { - Ciudadanía y multiculturalismo: balance y perspectivas para el siglo xxI } \\
\text { - ¿Sobre qué medita hoy la Teoría Política? } \\
\text { - Izquierdas y derechas contemporáneas: continuidad y cambio en el discurso político } \\
\text { - Democracia, ecología y sostenibilidad }\end{array}$ \\
\hline $\begin{array}{l}\text { La Laguna-Tenerife, } \\
2001\end{array}$ & $\begin{array}{l}\text { - Ciudadanía y políticas de integración en la Teoría Política: una perspectiva histórica } \\
\text { - Problemas de la ciudadanía en las democracias contemporáneas }\end{array}$ \\
\hline Barcelona, 2003 & $\begin{array}{l}\text { - Pluralismo y toleracia } \\
\text { - Las ideologías políticas en la Europa de hoy }\end{array}$ \\
\hline
\end{tabular}

Una primera lectura de estos grupos de trabajo nos proporciona de entrada dos informaciones importantes. En primer lugar, el enfoque que se practica: la TP en España es eminentemente una TP normativa. En segundo lugar, y en líneas generales, uno de los conceptos que más identifica la reflexión de la TP en España es, sin lugar a dudas, el de Democracia $^{40}$. La reflexión sustantiva sobre la democracia y su relación con los principales conceptos, tradiciones y desafíos de nuestra sociedad juega un papel motor evidente. En este marco global de reflexión, aparecen también cuatro líneas de trabajo que vertebran el universo de la TP en España:

\section{Ciudadanía.}

2. Formas de Multiculturalismo.

3. Tradiciones e ideologías políticas.

4. Teoría Política como disciplina.

40 Véase asimismo el interesante repaso de la TP que hace J. Pastor en la Unidad Didáctica de Introducción a la Ciencia Política titulada «Teorías y debates normativos» (J. Pastor, 2005) de la UNED. 
Si nos fijamos ahora en los temas de los principales artículos incluidos en el monográfico de la $R E C P\left(n .{ }^{\circ} 3,2000\right)$ editado por R. del Águila y dedicado a la $T P$, constatamos que la Democracia sigue siendo el elemento principal que articula la reflexión de la TP. Asimismo, si consideramos que cada artículo ilustra una línea de investigación en España, tenemos prácticamente una muestra de lo que se está trabajando actualmente. En la Introducción, R. del Águila apunta las principales discusiones:

1. Relación entre política y derecho para entender el reparto de poderes en sistemas democráticos.

2. Modelos de federalismo y sus problemas en relación a las democracias plurinacionales.

3. Relaciones entre nación y democracia.

4. Crisis del espacio público en las democracias contemporáneas.

Destacan también cuatro programas de investigación recientes:

5. Tradiciones de la ciudadanía.

6. Representación política.

7. Inmigración y justicia.

8. Teoría política verde.

Asimismo, la reciente edición de R. Máiz (comp., 2001) dedicada a autores contemporáneos que articulan el debate internacional muestra el vínculo que tiene la comunidad de la TP en España con los principales autores y ejes de reflexión que protagonizan la discusión contemporánea. R. Máiz (2001: 8) destaca en su Prefacio el debate liberalismo-comunitarismo, el multiculturalismo y nacionalismo liberal, la reformulación del igualitarismo, la renovación del republicanismo, la democracia participativa y de la deliberación, el posmodernismo y la crítica de los fundamentos, la teoría política de género, el pensamiento político verde, como temas prioritarios.

Por último, en esta breve presentación de la TP en España, no puedo dejar de citar lo que pienso también caracteriza la concepción que toda la comunidad comparte como función principal de la TP. La concepción que se tiene es sobre todo aplicada. Las palabras de R. del Águila en la Introducción de la monografía de la RECP ilustran lo que creo que todos los que practican la TP en España comparten: «La vocación de la teoría política no es (en realidad nunca fue) vivir al margen del mundo, sino intervenir en él» (R. del Águila, 2000: 8). 


\section{REFERENCIAS BIBLIOGRÁFICAS}

ACKERMAN, B. (1980): Social justice in the liberal State, New Haven: Yale University Press.

ÁGUILA, R. del (2000): «Política, derecho y razón de Estado», Revista Española de Ciencia Política, n. ${ }^{\circ}$ 3: 11-29.

- (ed.) (2000): Monográfico especial sobre la teoría política contemporánea, Revista Española de Ciencia Política, n. ${ }^{\circ} 3$.

ÁGUILA, R. del; VALLESPÍN, F., y otros (1998): La democracia en sus textos, Madrid: Alianza.

ALMOND, G. A. (1990): A discipline divided: schools and sects in political science, Londres: Sage Publications.

ANTÓN, J. (coord.) (2002): Las teorías políticas en el siglo XXI, Barcelona: Ariel.

ARATO, A., y COHEN, J. (1992): Civil Society and Political Theory, Cambridge, Mass.: MIT Press.

ARBLASTER, A. (1992): Democracy, Buckingham: Open University Press (Democracia, Madrid: Alianza).

AUBARELL, G., y ZAPATA BARRERO, R. (eds.) (2003): Inmigración y procesos de cambio: Europa y el Mediterráneo en el contexto global, Barcelona: Icaria.

BADER, V. (ed.) (1997): Citizenship and Exclusion, Londres: Macmillan Press.

BADILLO, P. (1998): Fundamentos de filosofía política, Madrid: Tecnos.

BALL, T. (1995): Reappraising political theory, Oxford: Clarendon Press.

BARBALET, J. M. (1988): Citizenship: rights, struggle and class inequality, M. Keynes: Open University Press.

BARBER, B. (1980): Political Theory (special isssue).

— (1984): Strong Democracy. Participatory politics for a new age, Berkeley, California: University of California Press.

BARRY, B. (1980): «The strange death of political philosophy», Government and Opposition, vol. 15/3-4: 276-288.

— (1989): Theories of Social Justice, Berkeley: University of California Press.

- (2001): «Teoría política: lo viejo y lo nuevo», en R. E. Goodin y H.-D. Klingemann (eds.), pp. 765-791.

BARRY, B., y GOODIN, R. E. (eds.) (1992): Free Movement, University Park, Pa.: Penn State University Press.

BARRY, N. P. (1995): An introduction to modern political theory, Nueva York: St. Martin's Press.

BAUBÖCK, R.; HELLER, A., y ZOLBERG, A. R. (eds.) (1996): The Challenge of diversity, Aldershot: Avebury, European Center Vienna V. 21.

BECK, U. (1992): Risk society, Londres: Sage.

BEETHAM, D. (1987): Bureaucracy, Milton Keynes: Open University Press.

- (1991): The legitimation of power, Londres: Macmillan.

BEITZ, C. (1979): Political theory and international relations, Princeton: Princeton University Press.

BELLAMY, R. (ed.) (1993): Theories and concepts of politics: an introduction, Manchester: Manchester University Press. 
BELLAMY, R. (1993): «Introduction: the demise and rise of political theory», en R. Bellamy (ed.), pp. 1-15.

BERLIN, I. (1962): «Does political theory still exist?», en P. Laslett y W. G. Runciman (eds.), pp. 1-33.

BEVIR, M. (1999): The logic of the history of ideas, Cambridge University Press.

BEYME, K. von (1994): Teoría política del siglo xx: de la modernidad a la postmodernidad, Madrid: Alianza.

- (2001): «Teoría política: teoría política empírica», en R. E. Goodin y H.-D. Klingemann (eds.), pp. 749-763.

BOBBIO, N. (1984): The future of Democracy, Minneapolis: University of Minnesota Press.

BRUBAKER, R. (ed.) (1989): Immigration and the politics of citizenship in Europe and North America, Boston: The University Press of America.

BUDGE (1993): «Direct democracy: setting appropiate terms of debate», en D. Held (ed.), pp. 136-55.

BURNHEIM, J. (1985): Is Democracy possible?, Oxford: Polity Press.

CARENS, J. (2000): Culture, citizenship, and community, Nueva York: Oxford University Press.

CLARK, L., y LANGE, L. (1979): The sexism of social and political theory, Toronto: University of Toronto Press.

COBBAN, A. (1953): «The decline of political theory», Political Science Quarterly, vol. 68/3: 321-337.

COHEN, J. (1983): Class and civil society, Amherst: University of Massachusetts Press.

CONNOLLY (1974): The terms of political discourse, Oxford: Blackwell.

DAHL, R. (1958): «Political Theory: truth and consequences», World Politics, 11: 89-102.

DAHL, R. A. (1989): Democracy and its critics, New Haven: Yale University Press.

DALLMAYR, F. (ed.) (1999): Border crossings: toward a comparative political theory, Boston: Lexington Books.

DIETZ, M. (1985): «Citizenship with a feminist face: the problem with maternal thinking», Political Theory, vol.13: 19-37.

DOBSON, A. (1997): Pensamiento político verde, Paidós.

DOGAN, M. (2001): «La ciencia política y las otras ciencias sociales», en R. E. Goodin y H.-D. Klingemann (eds.), pp. 150-196.

DRYSEK, J. (1990): Discursive democracy, Cambridge: Cambridge University Press.

DRYZEK, J. (1987): Rational ecology, Oxford: Basil Blackwell.

DUHERTY, B., y DE GEUS, M. (1996): Democracy and Green political thought, Londres: Routledge.

DWORKIN, R. (1977): Taking rights seriously, Cambridge, Mass.: Harvard University Press.

EASTON, D. (1953): The political system, Nueva York: Knopf.

— (1969): «The new revolution in political science», The American Political Science Review, vol. 63/4: 1051-1061.

ELSHTAIN, J. B. (1981): Public man, private woman, Princeton, NJ: Princeton University Press. 
ESPING-ANDERSEN, G. (1990): The three worlds of welfare capitalism, Oxford: Polity Press.

FARR, J., y SEIDELMAN, R. (eds.) (1993): Discipline and history: political science in the United States, Michigan: The University of Michigan Press.

FARR, J.; DRYZEK, J. S., y LEONARD, S. T. (eds.) (1995): Political science in history: research programs and political traditions, Cambridge: Cambridge University Press.

FINIFTER, A. W. (ed.) (1983): Political Science: The state of the discipline, Washington: American Political Science Association.

- (ed.) (1993): Political Science: The state of the discipline II, Washington: American Political Science Association.

FISHKIN, J. S. (1991): Deliberative Democracy, New Haven: Yale University Press.

FREEDEN, M. (1996): Ideologies and political theory, Oxford University Press.

GADAMER, H. G. (1992): Verdad y método, Salamanca: Ediciones Sígueme, 2 vols. (1986).

GALLIE (1955): «Essentially contested concepts», Proceedings of the Aristotelian Society, 56: 167-198.

GALSTON, W. A. (1980): Justice and the human good, Chicago-Londres: The University of Chicago Press.

— (1993): «Political theory in the 1980s: perplexity amidst diversity», en A. W. Finifter (ed.), pp. 27-53.

GOODIN, R. E. (1982): Political theory and public policy, Chicago: The University of Chicago Press.

— (1988): Reasons for welfare, Princeton, NJ: Princeton University Press.

— (1992): Green political theory, Cambridge: Polity Press.

GOODIN, R. E., y KLINGEMANN, H.-D. (2001): «Ciencia política: la discilpina», en R. E. Goodin y H.-D. Klingemann (eds.), pp. 21-81.

- (eds.) (2001): Nuevo manual de ciencia política, Madrid: Ediciones Istmo (A new handbook of political science, Oxford: Oxford University Press, 1996).

GOODIN, R. E., y PETTIT, P. H. (eds.) (1993): A companion to contemporary political philosophy, Oxford: Blackwell Publishers.

GRAY, J. (1994): Liberalismo, Madrid: Alianza.

GUNNELL, J. G. (1979): Political theory: tradition and interpretation, Cambridge, Mass.: Winthrop.

- (1982): «Interpretation and the history of political theory: apology and epistemology», The American Political Science Review, vol 76/2: 317-327.

GUNNELL, J. G. (1983): «Political theory: the evolution of a sub-field», en A. W. Finifter (ed.), pp. 3-45.

- (1986): Between philosophy and politics: the alienation of political theory, Amherst: The University of Massachusetts Press.

HABERMAS, J. (1989): El discurso filosófico de la modernidad, Madrid: Taurus (1. ${ }^{\text {a ed., } 1985) .}$

HAMLIN, A., y PETTIT, P. H. (eds.) (1989): The good polity narrative analysis of the State, Oxford: Basil Blackwell. 
HAMMAR, T. (1990): Democracy and the nation state: aliens, denizens and citizens in a world of international migration, Aldershot: Avebury.

HELD, D. (1987): Models of democracy, Oxford: Polity Press.

— (ed.) (1991): Political theory today, Cambridge: Polity Press.

- (1991): «Editor's introduction», en D. Held (ed.), pp. 1-21.

- (1991): Modelos de democracia, Madrid: Alianza

- (1998) Democracy and the global order, Cambridge: Polity Press (edición en castellano: La democracia y el orden global, Barcelona: Paidós, 1998).

HELD, D., y LEFTWICH, A. (1987): «¿Una disciplina de la política? », en A. Leftwich (comp.), pp. 254-290.

HINDESS, B. (1997): «The object of political theory», en A. Vincent (ed.), pp. 254-271.

HISRT, P. (1994): Associative democracy new forms of economic and social governance, Cambridge: Polity.

HONIG, B. (1993): Political theory and the displacement of politics, Ithaca, NY: Cornell University Press.

HONNETH, A. (1991): The critique of power, Cambridge: MIT Press.

KEANE, J. (1988): Democracy and Civil Society, Londres: Verso.

KELLEY, D. R. (1987): «Horizons of intellectual history: retrospect, circumspect, prospect», Journal of the History of Ideas, vol. 48, n. ${ }^{\circ}$ (Jan./March): 143-169.

KOSELLECK, R. (1993): Futuro pasado: para una semántica de los tiempos históricos, Barcelona: Paidós (1979) (Introducción, caps. V, X, XIV).

KUHN, T. S. (1962): The structure of scientific revolutions, Chicago: University of Chicago Press.

- (2000): The road since structure, Chicago: The University of Chicago Press (El camino desde la estructura, Barcelona: Paidós, 2002).

KYMLICKA, W. (1989): Liberalism, community and culture, Oxford: Clarendon Press.

- (1990): Contemporary political philosophy: an introduction, Oxford: Clarendon Press (Filosofía política contemporánea: una introducción, Barcelona: Ariel, 1995).

— (1995): Ciudadanía multicultural, Barcelona: Paidós.

LACAPRA, D. (1980): «Rethinking intellectual history and reading texts», History and Theory Studies in the Philosophy of History, vol. 19/3: 245-276; también, en D. Lacapra y S. L. Kaplan (eds.), cap. 2.

LACAPRA, D., y KAPLAN, S. L. (eds.) (1982): Modern European Intellectual History: reappraisals and new perspectives, Ithaca-London: Cornell University Press.

LASLETT, P. (ed.) (1956): Philosophy, politics and society, Oxford: Blackwell.

LIJPHART, A. (1977): Democracy in plural societies, New Haven: Yale University Press.

- (1984): Democracies: patterns of majoritarian and consensus government in twenty-one countries, New Haven: Yale University Press. 
LINDLEY, R. (1986): Autonomy, Londres: Macmillan.

LINZ, J., y STEPAN, A. (1978): The breakdown of democratic regimes, Baltimore: Johns Hopkins University Press.

LLOYD, J. (2002): «The end of multiculturalism», Newstatesman, mayo: 21-23.

LOCKYER, A. (1979): «"Traditions” as context in the history of political theory», Political Studies, vol. 27: 201-217.

LUCAS, J. (1996): Puertas que se cierran: Europa como fortaleza, Barcelona: Icaria.

LUHMAN (1993): Teoría política y Estado de bienestar, Madrid: Alianza.

MacINTYRE, A. (1981): After Virtue, Londres: Duckworth.

MacPHERSON, C. B. (1966): The real world of democracy, Oxford: Oxford University Press.

— (1973): Democratic theory: essays in retrieval, Oxford: Clarendon Press.

— (1977): The life and times of liberal democracy, Oxford: Oxford University Press.

- (1987): La democracia liberal y su época, Madrid: Alianza.

MÁIZ, R. (comp.) (2001): Teoría política contemporánea, Valencia: Tirant lo Blanch.

MÁIZ, R., y REQUEJO, F. (eds.) (2005): Democracy, Naturalism and Multiculturalism, Londres: Routledge.

MANSBRIDGE, J. J. (1980): Beyond adversary democracy, Nueva York: Basic Books.

McCARTHY, Th. (1978): The critical theory of Jürgen Habermas, Cambridge: MIT Press.

MENDUS, S. (1989): Toleration and the limits of liberalism, Londres: Macmillan.

MILLER, D. (1976): Social justice, Oxford: Clarendon Press.

— (1987): «Political theory», en J. Kuper (ed.), pp. 19-25.

— (1990): «The resurgence of political theory», Political Studies, vol. 38: 421-437.

- (1995): On Nationality, Nueva York: Oxford University Press (edición en castellano: Sobre la nacionalidad, Barcelona: Paidós, 1997).

MILLER, D., y SIEDENTOP, L. (eds.) (1983): The nature of political theory, Oxford: Clarendon Press.

MODOOD, T.; TRIANDAFYLLIDOU, A., y ZAPATA BARRERO, R. (eds.) (2005): Multiculturalism, Muslimsand Citizenship: A European approach, Londres: Routledge.

MOUFFE, Ch. (ed.) (1992): Dimensions of radical democracy: pluralism, citizenship, community, Londres: Verso.

- (1993): The return of the political, Londres: Verso.

MULHALL, S., y SWIFT, A. (1992): Liberals and communitarians, Oxford: Blackwell.

NEDERMAN, C. J., y ELLIOT, P. M. (1983): «Bibliography: articles in political theory, 1979-1981», Political Theory, vol. 11/2: 273-317.

NELSON, J. S. (ed.) (1986): Tradition, Interpretation, and Science: political theory in the American Academy, Albany: State University of New York Press. 
NICHOLSON, L. (1986): Gender and history, Nueva York: Columbia University Press.

NOZICK, R. (1974): Anarchy, State, and Utopia, Oxford: Blackwell (versión castellana: R. Tamayo, Anarquía, Estado y Utopía, México: Fondo de Cultura Económica, 1988).

O'SULLIVAN, N. (2000): «Introduction», en N. O’Sullivan (ed.), pp. 1-16.

- (ed.) (2000): Political Theory in Transition, Londres: Routledge.

OKIN, S. M. (1989): Justice, gender and the family, Nueva York: Basic Books.

PALONEN, K. (2002): «The history of concepts as a style of political theorizing», European Journal of Political Theory, 1 (1): 91-106.

PAREKH, B. (1968): «The nature of political philosophy», en P. King y B. Parekh (eds.), pp. 153-207.

- (2000): «Theorizing political theory», en N. O’Sullivan (ed.), cap. 13, pp. 242-259.

- (2001): «Teoría política: tradiciones en filosofía política», en R. E. Goodin y H.-D. Klingemann (eds.), pp. 727-747.

PAREL, A. J., y KEITH, R. C. (eds.) (1992): Comparative political philosophy, Nueva Delhi: Sage Publications.

PASTOR, J. (2005): «Teorías y debates normativos», en Unidad Didáctica I de Introducción a la Ciencia Política, Madrid: UNED [http://www.uned.es.dcpa/Asignaturas/Intropol/UDI/Intropol_UD_I_capitulo_tres.pdf], junio.

PATEMAN, C. (1970): Participation and democratic theory, Cambridge University Press.

PETTIT, Ph. (1991): Contemporary political theory, Nueva York: Maxwell MacMillan International Editions.

— (1999): Republicanismo, Barcelona: Paidós.

PHILLIPS, A. (1991): Engendering democracy, Pennsylvania State University.

— (1993): Democracy and difference, Cambridge: Polity Press.

PITKIN, H. F. (1984): Fortune is a woman, Berkeley: University of California Press.

PLAMENATZ, J. (1960): «The use of political theory», Political Studies, vol. 8.

POCOCK, J. G. A. (1981): «The reconstruction of discourse: towards the historiography of political thought», Modern Language Notes, vol. 96, n. ${ }^{\circ} 5$ (december): 959-980.

- (1982): «The history of political thought: a methodology enquiry», en P. Laslett y W. G. Runciman (eds.), Philosophy, politics, and society (2 ${ }^{\text {nd }}$ Series), Oxford, cap. 9, pp. 183-202.

- (1985): Virtue, commerce, and history, Cambridge: Cambridge University Press («Introduction: the state of the art», pp. 1-34.)

PUTNAM, R. (1993): Making democracy work, Princeton: Princeton University Press.

RAWLS, J. (1971): A theory of justice, Cambridge: Harvard University Press.

— (1993): Political liberalism, Nueva York: Columbia University Press.

RAZ, J. (1986): The morality of freedom, Oxford: Clarendon.

REQUEJO, F. (ed.) (2001): Democracy and national pluralism, Routledge (trad. en Ariel, 2002). 
REQUEJO, F. (2005): Multinational Federalism \& Value Pluralism: The Spanish Case, Londres: Routledge.

RICHTER, M. (1995): The history of political and social concepts, Cambridge: Oxford University Press.

RORTY, R. (1989): Contingency, irony, and solidarity, Cambridge: Cambridge University Press.

RORTY, R. (1991): Objectivity, relativism, and truth: philosophical papers, Cambridge: Cambridge University Press, vol. 1 .

SABINE, G. H. (1939): «What is a political theory?», The Journal of Politics, vol. 1/1: 1-16.

SABINE, G. H., y THORSON, T. (1937): A History of Political Theory, Nueva York: Holt, Reinehart and Winston (Historia de la teoría política, México: FCE, 1992).

SANDEL, M. (1982): Liberalism and the limits of justice, Cambridge: Cambridge University Press.

SAXONHOUSE, A. W. (1993): «Texts and canons: the status of the "great books" in political theory», en A. W. Finifter (ed.), pp. 3-26.

SCHMITTER, P., y LEHMBRUCH, G. (eds.) (1979): Trends toward corporate intermediation, Beverly Hills: Sage.

SCHMITTER, P.; O'DONNELL, G., y WHITEHEAD, L. (1986): Transitions from authoritarian rule, Baltimore: John Hopkins University Press.

SCHWARTZ, W. F. (1995) (ed.): Justice in immigration, Cambridge: Cambridge University Press.

SELF, P. (1988): Political theories of modern government, its role and reform, Londres: Unwin Hyman.

SEN, A. (1992): Inequality reexamined, Oxford: Clarendon Press.

SHANLEY, M. L., y PATEMAN, C. (eds.) (1991): Feminist interpretations and political theory, Cambridge University Press.

SHAPIRO, I. (2002): «Problems, methods, and theories in the study of politics, or what's wrong with political science and what to do about it», Political Theory, 30/4: 596-619.

SKINNER, Q. (1969): «Meaning and Understanding in the history of ideas», History and Theory, vol. 8: 3-53.

- (1970): “Social Meaning” and the explanation of social action», en P. Laslett, W. C. Runciman y Q. Skinner, Philosophy, politics, society, Series 4, Oxford: Blackwell, pp. 136-157.

- (1974): «Some problems in the analysis of political thought and action», Political Theory, vol. 2, n. ${ }^{\circ}$ 3: $277-303$.

TARLTON, Ch. D. (1973): «Historicity, meaning, and revisionism in the study of political thought», History and Theory, vol. 12: 311-328.

TAYLOR, Ch. (1983): «Political theory and practice», en Ch. Taylor (ed.), pp. 61-85.

- (ed.) (1983): Social theory and political practice, Oxford: Clarendon Press.

- (1985): Philosophical papers, vol. I: Human agency and language, y vol. II: Philosophy and human sciences, Cambridge: Cambridge University Press.

- (1992): Multiculturalism and «the politics of recognition», Princeton (EUA): Princeton University Press (edición en castellano: El multiculturalismo y «la política del reconocimiento», México: Fondo de Cultura Económica, 1993). 
VALLESPÍN, F. (coord.) (1990): Aspectos metodológicos en la Historia de la Teoría Política, vol. 1, pp. 19-39.

- (1992): «El pensamiento en la historia», Revista del CECs, 13 (septiembre-diciembre): 51-178.

— (coord.) (1992-95): Historia de la Teoría Política, 6 vols., Madrid: Alianza

VINCENT, A. (1997): «Introduction», en A. Vincent (ed.), pp. 1-27.

VINCENT, A. (ed.) (1997): Political theory. Tradition and diversity, Cambridge: Cambridge University Press.

VV.AA. (1990): «The Nature of Contemporary Political Science: a roundtable discussion», Political Science and Politics, vol. $23(1)$.

WALKER, R. B. J. (1993): Inside/outside: international relations as political theory, Cambridge University Press.

WALZER, M. (1970): Obligations: essays on disobedience war and citizenship, Cambridge, Mass.: Harvard University Press.

— (1983): Spheres of Justice: a defense of pluralism and equality, Nueva York: Basic Books.

— (ed.) (1989): «The state of political theory», Dissent, monográfico especial verano.

WATSON, C. W. (2000): Multiculturalism, Buckingham: Open University Press.

WELDON, T. D. (1953): The Vocabulary of Politics, Baltimore: Penguin Books (caps.1-2)

WHITE, S. (1988): The recent work of Jürgen Habermas: Reason, justice and modernity, Cambridge: Cambridge University Press.

- (1991) Politcal Theory and postmodernism, Cambridge: Cambridge University Press.

— (ed.) (2002): «What is political theory?» (special issue), Political Theory, 30/4.

— (2002): «Pluralism, platitudes, and paradoxes: fifty years of western political thought», Political Theory, 30/4: $472-481$.

WILTSHIRE, K. (ed.) (2001): «Management of social transformations» (special issue), International Political Science Review, vol. 22/1, enero.

WOLFF, R. P. (1970): In defense of anarquism, Nueva York: Harper.

WOLIN, S. (1960): Política y perspectiva, Buenos Aires: Amorrortu.

— (1968): «Paradigms and political theories», en P. King y B. C. Parekh (eds.), pp. 125-152.

- (1969): «Political theory as a vocation», The American Political Science Review, vol. 63/4: 1062-1082.

YOUNG, I. M. (1990): Justice and the politics of difference, Princeton, NJ: Princeton University Press.

- (2000): Inclusion and democracy, Oxford: Oxford University Press.

- (2001): «Teoría política: una visión general», en R. E. Goodin y H.-D. Klingemann (eds.), pp. 693-725.

ZAPATA BARRERO, R. (coord.) (2001a): «Ciudadanía e interculturalidad: balance y persectivas para el siglo XXI», monográfico de la Revista Anthropos, febrero-marzo: 191.

- (2001b): Ciudadanía, democracia y pluralismo cultural: hacia un nuevo contrato social, Barcelona: Anthropos (Libros de la Revista Anthropos). 
ZAPATA BARRERO, R. (2002): El turno de los inmigrantes: esferas de justicia y políticas de acomodación, Imserso, Ministerio de Trabajo y Seguridad Social (traducción catalana: L'hora dels immigrants: esferes de justícia i polítiques d'acomodació, Barcelona: Proa).

- (2004a): Multiculturalidad e inmigración, Madrid: Síntesis.

- (2004b): Inmigración, Innovación Política y cultura de acomodación en España: Un análisis comparativo entre Andalucía, Cataluña, la Comunidad de Madrid, y el Gobierno Central, Ed. Cidob.

- (ed.) (2005): Manual de Conceptos Políticos en el contexto de España, Madrid: Ed. Síntesis (en prensa).

\section{ABSTRACT}

The rapid changes of parameters we have experienced in recent years are confronting social science researchers with new scenarios and perspectives. These situations are so new that the conceptual task of enquiring into how to approach these new contexts is becoming an imperative for the innovative dynamics of the discipline in itself. There is an increasingly widely-held conviction that the theoretical reflections and political decisions that are made here and now are of vital importance, as what is being designed is the political society of the future. Within this framework, political theory (PT) as a critical activity has fertile soil that is unprecedented in recent history. We have to pose two questions that are linked with one another: What is its function in relation to this reality of social and political instability? What are the instruments (concepts, approaches, etc.) it can provide us with for the analysis of these structural changes? I will articulate my reasoning into six chapters. In the first, I present the first elements for initiating a reflection on PT as it is today. In the second, I consider which is the viewpoint from which the political theoretician interprets changing reality, centring particularly on its unity of analysis and basic aims. Chapter three is historical. I review the main stages of PT, focussing on the last in accordance with my central thread: the analysis of the contexts of social and political changes. I consider then, in the fourth chapter, which are the main research programs that contribute at present to the study of these structural changes. In order to complete this argument, in chapter five I go deeper into the main current approaches that exist for analysing these changes, which determine different forms of understanding the activity of PT itself. Finally, in the sixth and last chapter, I return to the current meaning of PT, indicating its main challenges and expectations, as manager of political innovation. I end with some final reflections to leave this research program open. In a appendix, I present some arguments on PT in Spain, as a basis for future research.

Key words: Political Theory, Historical Evolution, Research Programmes, Processes of Change, Political Innovation.. 